\title{
Multistability and Instability of Competitive Neural Networks with Mexican-Hat-Type Activation Functions
}

\author{
Xiaobing Nie,, Jinde Cao, ${ }^{1,3}$ and Shumin Fei ${ }^{2}$ \\ ${ }^{1}$ Department of Mathematics, and Research Center for Complex Systems and Network Sciences, Southeast University, \\ Nanjing 210096, China \\ ${ }^{2}$ School of Automation, Southeast University, Nanjing 210096, China \\ ${ }^{3}$ Department of Mathematics, Faculty of Science, King Abdulaziz University, Jeddah 21589, Saudi Arabia
}

Correspondence should be addressed to Xiaobing Nie; xbnie@seu.edu.cn

Received 2 January 2014; Accepted 9 April 2014; Published 6 May 2014

Academic Editor: Weinian Zhang

Copyright (C) 2014 Xiaobing Nie et al. This is an open access article distributed under the Creative Commons Attribution License, which permits unrestricted use, distribution, and reproduction in any medium, provided the original work is properly cited.

\begin{abstract}
We investigate the existence and dynamical behaviors of multiple equilibria for competitive neural networks with a class of general Mexican-hat-type activation functions. The Mexican-hat-type activation functions are not monotonously increasing, and the structure of neural networks with Mexican-hat-type activation functions is totally different from those with sigmoidal activation functions or nondecreasing saturated activation functions, which have been employed extensively in previous multistability papers. By tracking the dynamics of each state component and applying fixed point theorem and analysis method, some sufficient conditions are presented to study the multistability and instability, including the total number of equilibria, their locations, and local stability and instability. The obtained results extend and improve the very recent works. Two illustrative examples with their simulations are given to verify the theoretical analysis.
\end{abstract}

\section{Introduction}

In the past decades, some famous neural network models, including Hopfield neural networks, cellular neural networks, Cohen-Grossberg neural networks, and bidirectional associative memory neural networks, had been proposed in order to solve some practical problems. It should be mentioned that in the above network models only the neuron activity is taken into consideration. That is, there exists only one type of variables, the state variables of the neurons in these models. However, in a dynamical network, the synaptic weights also vary with respect to time due to the learning process, and the variation of connection weights may have influences on the dynamics of neural network. Competitive neural networks (CNNs) constitute an important class of neural networks, which model the dynamics of cortical cognitive maps with unsupervised synaptic modifications. In this model, there are two types of state variables: that of the short-term memory (STM) describing the fast neural activity and that of long-term memory (LTM) describing the slow unsupervised synaptic modifications. The CNNs can be written in the following form:

$$
\begin{aligned}
\operatorname{STM}: \dot{x}_{i}(t)= & -d_{i} x_{i}(t)+\sum_{j=1}^{N} a_{i j} f_{j}\left(x_{j}(t)\right) \\
& +B_{i} \sum_{j=1}^{P} m_{i j}(t) y_{j}+I_{i}, \\
\operatorname{LTM}: \dot{m}_{i j}(t)= & -\alpha_{i} m_{i j}(t)+y_{j} \beta_{i} f_{i}\left(x_{i}(t)\right),
\end{aligned}
$$

where $i=1,2, \ldots, N, j=1,2, \ldots, P, x_{i}(t)$ is the neuron current activity level, $f_{j}\left(x_{j}(t)\right)$ is the output of neurons, $m_{i j}(t)$ is the synaptic efficiency, $y_{j}$ is the constant external stimulus, $a_{i j}$ represents the connection weight between the $i$ th neuron and the $j$ th neuron, $B_{i}$ is the strength of the external stimulus, $I_{i}$ is the constant input, and $\alpha_{i}>0$ and $\beta_{i} \geq 0$ denote disposable scaling constants. 
After setting $S_{i}(t)=\sum_{j=1}^{P} m_{i j}(t) y_{j}=\mathbf{y}^{T} \mathbf{m}_{i}(t)$, where $\mathbf{y}=$ $\left(y_{1}, y_{2}, \ldots, y_{P}\right)^{T}$ and $\mathbf{m}_{i}(t)=\left(m_{i 1}(t), m_{i 2}(t), \ldots, m_{i P}(t)\right)^{T}$ and assuming the input stimulus $\mathbf{y}$ to be normalized with unit magnitude $|\mathbf{y}|^{2}=y_{1}^{2}+\cdots+y_{P}^{2}=1$, then the above networks are simplified as

$$
\begin{aligned}
& \dot{x}_{i}(t)=-d_{i} x_{i}(t)+\sum_{j=1}^{N} a_{i j} f_{j}\left(x_{j}(t)\right)+B_{i} S_{i}(t)+I_{i}, \\
& \dot{S}_{i}(t)=-\alpha_{i} S_{i}(t)+\beta_{i} f_{i}\left(x_{i}(t)\right), \quad i=1,2, \ldots, N .
\end{aligned}
$$

The qualitative analysis of neural dynamics plays an important role in the design of practical neural networks. To solve problems of optimization and signal processing, neural networks have to be designed in such a way that, for a given external input, they exhibit only one globally stable state (i.e., monostability). This matter has been treated in [1-7]. On the other hand, if neural networks are used to analyze associative memories, the coexistence of multiple locally stable equilibria or periodic orbits is required (i.e., multistability or multiperiodicity), since the addressable memories or patterns are stored as stable equilibria or stable periodic orbits. In monostability analysis, the objective is to derive conditions that guarantee that each network contains only one steady state, and all the trajectories of the network converge to it, whereas in multistability analysis, the networks are allowed to have multiple equilibria or periodic orbits (stable or unstable). In general, the usual global stability conditions are not adequately applicable to multistable networks.

Recently, the multistability or multiperiodicity of neural networks has attracted the attention of many researchers. In $[8,9]$, based on decomposition of state space, the authors investigated the multistability of delayed Hopfield neural networks and showed that the $n$-neuron neural networks can have $2^{n}$ stable orbits located in $2^{n}$ subsets of $\mathbb{R}^{n}$. Cao et al. [10] extended the above method to the Cohen-Grossberg neural networks with nondecreasing saturated activation functions with two corner points. In $[11,12]$, the multistability of almostperiodic solution in delayed neural networks was studied. Kaslik and Sivasundaram $[13,14]$ firstly revealed the effect of impulse on the multistability of neural networks. In [1517], high-order synaptic connectivity was introduced into neural networks and the multistability and multiperiodicity were considered, respectively, for high-order neural networks based on decomposition of state space, Cauchy convergence principle, and inequality technique. In [18-22], the authors indicated that under some conditions, there exist $3^{n}$ equilibria for the $n$-neuron neural networks and $2^{n}$ of which are locally exponentially stable. In [23], the Hopfield neural networks with nondecreasing piecewise linear activation functions with $2 r$ corner points were considered. It was proved that under some conditions, the $n$-neuron neural networks can have and only have $(2 r+1)^{n}$ equilibria, $(r+1)^{n}$ of which are locally exponentially stable and others are unstable. In [24], the multistability of neural networks with $k+m$ step stair activation functions was discussed based on an appropriate partition of the $n$-dimensional state space. It was shown that the $n$-neuron neural networks can have $(2 k+2 m-1)^{n}$ equilibria, $(k+m)^{n}$ of which are locally exponentially stable.

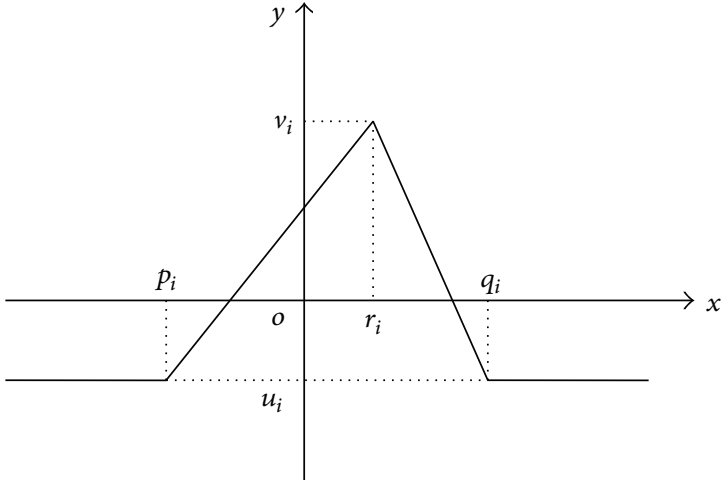

FIgURE 1: Mexican-hat-type activation functions (3).

In particular, the case of $k=m$ was previously discussed in [25]. For more references, see [26-32] and references therein.

It is well known that the type of activation functions plays a very important role in the multistability analysis of neural networks. In the abovementioned and most existing works, the activation functions employed in multistability analysis were mainly focused on sigmoidal activation functions and nondecreasing saturated activation functions, which are all monotonously increasing. In this paper, we will consider a class of continuous Mexican-hat-type activation functions, which are defined as follows (see Figure 1):

$$
f_{i}(x)= \begin{cases}u_{i}, & -\infty<x<p_{i} \\ l_{i, 1} x+c_{i, 1}, & p_{i} \leq x \leq r_{i} \\ l_{i, 2} x+c_{i, 2}, & r_{i}<x \leq q_{i}, \\ u_{i}, & q_{i}<x<+\infty\end{cases}
$$

where $p_{i}, r_{i}, q_{i}, u_{i}, l_{i, 1}, l_{i, 2}, c_{i, 1}, c_{i, 2}$ are constants with $-\infty<$ $p_{i}<r_{i}<q_{i}<+\infty, l_{i, 1}>0$, and $l_{i, 2}<0, i=1,2, \ldots, N$. In particular, when $p_{i}=-1, r_{i}=1, q_{i}=3, u_{i}=-1$, $l_{i, 1}=1, l_{i, 2}=-1, c_{i, 1}=0$, and $c_{i, 2}=2(i=1,2, \ldots, N)$, the above activation functions $f_{i}$ reduce to the following special activation functions employed in [33]:

$$
f_{i}(x)= \begin{cases}-1, & -\infty<x<-1, \\ x, & -1 \leq x \leq 1, \\ -x+2, & 1<x \leq 3 \\ -1, & 3<x<+\infty .\end{cases}
$$

It is necessary to point out that the Mexican-hat-type activation functions are not monotonously increasing, which are totally different from sigmoidal activation functions and nondecreasing saturated activation functions. Hence, the results and methods mentioned above cannot be applied to neural networks with activation functions (3). Very recently, the multistability and instability of Hopfield neural networks with activation functions (4) were studied in [33]. Inspired by [33], in this paper, we will investigate the multistability and instability of CNNs with activation functions (3). It should be noted that the structure of CNNs differs from and is more complex than that in [33]. Moreover, the activation functions 
(3) employed in this paper are more general than activation functions (4). More precisely, the contributions of this paper are three-fold as follows.

Firstly, we define four index subsets and present sufficient condition under which the CNNs with activation functions (3) have multiple equilibria, by tracking the dynamics of each state component and applying fixed point theorem. The index subsets are defined in terms of maximum and minimum values, which are different from and less restrictive than those given in [33]. Furthermore, we discuss the exact existence of equilibria for CNNs.

Secondly, based on some analysis method, we analyze the dynamical behaviors of each equilibrium point for CNNs, including local stability and instability. The dynamical behaviors of such system are much more complex than those of Hopfield neural networks considered in [33], due to the complexity of the networks structure and generality of activation functions.

Thirdly, specializing the model and activation functions to those in [33], we show that the obtained results extend and improve the very recent works in [33].

Finally, two examples with their simulations are given to verify and illustrate the validity of the obtained results.

\section{Main Results}

Firstly, we define the four index subsets as follows:

$$
\begin{aligned}
& \mathbb{N}_{1}=\left\{i: I_{i}<-\max \left\{-d_{i} p_{i}+a_{i i} f_{i}\left(p_{i}\right),-d_{i} r_{i}+a_{i i} f_{i}\left(r_{i}\right)\right\}\right. \\
& -\sum_{j \neq i, j=1}^{N} \max \left\{a_{i j} u_{j}, a_{i j} v_{j}\right\} \\
& \left.-\max \left\{\alpha_{i}^{-1} \beta_{i} u_{i} B_{i}, \alpha_{i}^{-1} \beta_{i} v_{i} B_{i}\right\}\right\}, \\
& \mathbb{N}_{2}=\left\{i: d_{i} r_{i}-a_{i i} f_{i}\left(r_{i}\right)-\sum_{j \neq i, j=1}^{N} \min \left\{a_{i j} u_{j}, a_{i j} v_{j}\right\}\right. \\
& -\min \left\{\alpha_{i}^{-1} \beta_{i} u_{i} B_{i}, \alpha_{i}^{-1} \beta_{i} v_{i} B_{i}\right\} \\
& <I_{i}<d_{i} p_{i}-a_{i i} f_{i}\left(p_{i}\right)-\sum_{j \neq i, j=1}^{N} \max \left\{a_{i j} u_{j}, a_{i j} v_{j}\right\} \\
& \left.-\max \left\{\alpha_{i}^{-1} \beta_{i} u_{i} B_{i}, \alpha_{i}^{-1} \beta_{i} v_{i} B_{i}\right\}\right\}, \\
& \mathbb{N}_{3}=\left\{i:-\min \left\{-d_{i} p_{i}+a_{i i} f_{i}\left(p_{i}\right),-d_{i} r_{i}+a_{i i} f_{i}\left(r_{i}\right)\right\}\right. \\
& -\sum_{j \neq i, j=1}^{N} \min \left\{a_{i j} u_{j}, a_{i j} v_{j}\right\}
\end{aligned}
$$

$$
\begin{aligned}
& -\min \left\{\alpha_{i}^{-1} \beta_{i} u_{i} B_{i}, \alpha_{i}^{-1} \beta_{i} v_{i} B_{i}\right\}<I_{i}<d_{i} q_{i} \\
& -a_{i i} f_{i}\left(q_{i}\right)-\sum_{j \neq i, j=1}^{N} \max \left\{a_{i j} u_{j}, a_{i j} v_{j}\right\} \\
& \left.-\max \left\{\alpha_{i}^{-1} \beta_{i} u_{i} B_{i}, \alpha_{i}^{-1} \beta_{i} v_{i} B_{i}\right\}\right\} \\
& \mathbb{N}_{4}=\left\{i: I_{i}>-\min \left\{-d_{i} q_{i}+a_{i i} f_{i}\left(q_{i}\right),-d_{i} r_{i}+a_{i i} f_{i}\left(r_{i}\right)\right\}\right. \\
& -\sum_{j \neq i, j=1}^{N} \min \left\{a_{i j} u_{j}, a_{i j} v_{j}\right\} \\
& \left.-\min \left\{\alpha_{i}^{-1} \beta_{i} u_{i} B_{i}, \alpha_{i}^{-1} \beta_{i} v_{i} B_{i}\right\}\right\} \text {, }
\end{aligned}
$$

where $v_{i}=f_{i}\left(r_{i}\right)$. It is easy to see that $u_{i} \leq f_{i}(x) \leq v_{i}$ for $x \in \mathbb{R}$.

Remark 1. In this paper, the index subsets are defined in terms of maximum and minimum values, which are different from those given in [33], where they are defined in terms of absolute values. In general, our conditions are less restrictive, which have been shown in [16].

Remark 2. The inequality $d_{i}-\left(a_{i i}+\alpha_{i}^{-1} \beta_{i} B_{i}\right) l_{i, 1}<0$ holds for all $i \in \mathbb{N}_{2}$.

Proof. By the definition of index subset $\mathbb{N}_{2}, u_{i}=f_{i}\left(p_{i}\right)$ and $v_{i}=f_{i}\left(r_{i}\right)$, we obtain

$$
\begin{aligned}
& -d_{i} p_{i}+\left(a_{i i}+\alpha_{i}^{-1} \beta_{i} B_{i}\right) f_{i}\left(p_{i}\right) \\
& +\sum_{j \neq i, j=1}^{N} \max \left\{a_{i j} u_{j}, a_{i j} v_{j}\right\}+I_{i}<0, \\
& -d_{i} r_{i}+\left(a_{i i}+\alpha_{i}^{-1} \beta_{i} B_{i}\right) f_{i}\left(r_{i}\right) \\
& +\sum_{j \neq i, j=1}^{N} \min \left\{a_{i j} u_{j}, a_{i j} v_{j}\right\}+I_{i}>0 .
\end{aligned}
$$

It follows from (6) and (7) that

$$
\begin{aligned}
-d_{i} p_{i} & +\left(a_{i i}+\alpha_{i}^{-1} \beta_{i} B_{i}\right) f_{i}\left(p_{i}\right) \\
<-d_{i} r_{i} & +\left(a_{i i}+\alpha_{i}^{-1} \beta_{i} B_{i}\right) f_{i}\left(r_{i}\right) .
\end{aligned}
$$

Noting that $p_{i}<r_{i}$ and substituting $f_{i}\left(p_{i}\right)=l_{i, 1} p_{i}+c_{i, 1}$ and $f_{i}\left(r_{i}\right)=l_{i, 1} r_{i}+c_{i, 1}$ into (8), we can derive that $d_{i}-\left(a_{i i}+\right.$ $\left.\alpha_{i}^{-1} \beta_{i} B_{i}\right) l_{i, 1}<0\left(i \in \mathbb{N}_{2}\right)$.

Remark 3. The inequality $d_{i}-\left(a_{i i}+\alpha_{i}^{-1} \beta_{i} B_{i}\right) l_{i, 2}>0$ holds for all $i \in \mathbb{N}_{2} \cup \mathbb{N}_{3}$. 
Proof. From Remark 2, we get $a_{i i}+\alpha_{i}^{-1} \beta_{i} B_{i}>0\left(i \in \mathbb{N}_{2}\right)$. Thus, inequality $d_{i}-\left(a_{i i}+\alpha_{i}^{-1} \beta_{i} B_{i}\right) l_{i, 2}>0$ holds for all $i \in \mathbb{N}_{2}$, due to $l_{i, 2}<0$.

By the definition of index subset $\mathbb{N}_{3}$ and equalities $u_{i}=$ $f_{i}\left(q_{i}\right), v_{i}=f_{i}\left(r_{i}\right)$, we get

$$
\begin{aligned}
\min & \left\{-d_{i} p_{i}+a_{i i} f_{i}\left(p_{i}\right),-d_{i} r_{i}+a_{i i} f_{i}\left(r_{i}\right)\right\} \\
& +\alpha_{i}^{-1} \beta_{i} v_{i} B_{i}>-d_{i} q_{i}+a_{i i} f_{i}\left(q_{i}\right)+\alpha_{i}^{-1} \beta_{i} u_{i} B_{i},
\end{aligned}
$$

which implies that $-d_{i} r_{i}+\left(a_{i i}+\alpha_{i}^{-1} \beta_{i} B_{i}\right) f_{i}\left(r_{i}\right)>-d_{i} q_{i}+\left(a_{i i}+\right.$ $\left.\alpha_{i}^{-1} \beta_{i} B_{i}\right) f_{i}\left(q_{i}\right)$. By using equalities $f_{i}\left(r_{i}\right)=l_{i, 2} r_{i}+c_{i, 2}, f_{i}\left(q_{i}\right)=$ $l_{i, 2} q_{i}+c_{i, 2}$ and noting that $r_{i}<q_{i}$, the inequality $d_{i}-\left(a_{i i}+\right.$ $\left.\alpha_{i}^{-1} \beta_{i} B_{i}\right) l_{i, 2}>0\left(i \in \mathbb{N}_{3}\right)$ can be proved easily.

It follows from the second equation of system (2) that

$$
-\alpha_{i} S_{i}(t)+\beta_{i} u_{i} \leq \dot{S}_{i}(t) \leq-\alpha_{i} S_{i}(t)+\beta_{i} v_{i},
$$

which leads to

$$
\begin{aligned}
& \alpha_{i}^{-1} \beta_{i} u_{i}+\left(S_{i}(0)-\alpha_{i}^{-1} \beta_{i} u_{i}\right) e^{-\alpha_{i} t} \\
& \quad \leq S_{i}(t) \leq \alpha_{i}^{-1} \beta_{i} v_{i}+\left(S_{i}(0)-\alpha_{i}^{-1} \beta_{i} v_{i}\right) e^{-\alpha_{i} t} .
\end{aligned}
$$

Therefore, $S_{i}(0) \in\left[\alpha_{i}^{-1} \beta_{i} u_{i}, \alpha_{i}^{-1} \beta_{i} v_{i}\right]$ always implies that $S_{i}(t) \in\left[\alpha_{i}^{-1} \beta_{i} u_{i}, \alpha_{i}^{-1} \beta_{i} v_{i}\right]$. That is, if $S(0) \epsilon$ $\prod_{i=1}^{N}\left[\alpha_{i}^{-1} \beta_{i} u_{i}, \alpha_{i}^{-1} \beta_{i} v_{i}\right]$, then the solution $S(t ; S(0))$ will stay in $\prod_{i=1}^{N}\left[\alpha_{i}^{-1} \beta_{i} u_{i}, \alpha_{i}^{-1} \beta_{i} v_{i}\right]$ for all $t \geq 0$.

Let $\left(x^{T}(t), S^{T}(t)\right)^{T}$ be a solution of system (2) with initial state $\left(x^{T}(0), S^{T}(0)\right)^{T} \in \mathbb{R}^{N} \times \prod_{i=1}^{N}\left[\alpha_{i}^{-1} \beta_{i} u_{i}, \alpha_{i}^{-1} \beta_{i} v_{i}\right]$. In the following, we will discuss the dynamics of state components $x_{i}(t)$ for $i \in \mathbb{N}_{i}(i=1,2,3,4)$, respectively.

Lemma 4. All the state components $x_{i}(t), i \in \mathbb{N}_{1}$, will flow to the interval $\left(-\infty, p_{i}\right]$ when $t$ tends to $+\infty$.

Proof. According to the different location of $x_{i}(0)$, there are two cases for us to discuss.

Case (i). Consider $x_{i}(0) \in\left(-\infty, p_{i}\right]$. In this case, if there exists some $t^{*} \geq 0$ such that $x_{i}\left(t^{*}\right)=p_{i}, x_{i}(t) \leq p_{i}$ for $0 \leq t \leq t^{*}$, then it follows from system (2) and the definition of $\mathbb{N}_{1}$ that

$$
\begin{aligned}
\dot{x}_{i}\left(t^{*}\right)= & -d_{i} x_{i}\left(t^{*}\right)+a_{i i} f_{i}\left(x_{i}\left(t^{*}\right)\right) \\
& +\sum_{j \neq i, j=1}^{N} a_{i j} f_{j}\left(x_{j}\left(t^{*}\right)\right)+B_{i} S_{i}\left(t^{*}\right)+I_{i} \\
\leq & -d_{i} p_{i}+a_{i i} f_{i}\left(p_{i}\right)+\sum_{j \neq i, j=1}^{N} \max \left\{a_{i j} u_{j}, a_{i j} v_{j}\right\} \\
& +\max \left\{\alpha_{i}^{-1} \beta_{i} u_{i} B_{i}, \alpha_{i}^{-1} \beta_{i} v_{i} B_{i}\right\}+I_{i}<0 .
\end{aligned}
$$

Hence, $x_{i}(t)$ would never get out of $\left(-\infty, p_{i}\right]$. Similarly, we can also conclude that once $x_{i}\left(T_{0}\right) \in\left(-\infty, p_{i}\right]$ for some $T_{0}>$ 0 , then $x_{i}(t)$ would never escape from $\left(-\infty, p_{i}\right]$ for all $t \geq T_{0}$.

Case (ii). Consider $x_{i}(0) \in\left(p_{i},+\infty\right)$. In this case, we claim that $x_{i}(t)$ would monotonously decrease until it reaches the interval $\left(-\infty, p_{i}\right]$ in some finite time.
In fact, when $x_{i}(t) \in\left(q_{i},+\infty\right)$, noting that the definition of $\mathbb{N}_{1}$ and $f_{i}\left(p_{i}\right)=f_{i}\left(q_{i}\right)$, we obtain

$$
\begin{aligned}
\dot{x}_{i}(t)= & -d_{i} x_{i}(t)+a_{i i} f_{i}\left(x_{i}(t)\right) \\
& +\sum_{j \neq i, j=1}^{N} a_{i j} f_{j}\left(x_{j}(t)\right)+B_{i} S_{i}(t)+I_{i} \\
\leq & -d_{i} q_{i}+a_{i i} f_{i}\left(q_{i}\right)+\sum_{j \neq i, j=1}^{N} a_{i j} f_{j}\left(x_{j}(t)\right)+B_{i} S_{i}(t)+I_{i} \\
\leq & -d_{i} p_{i}+a_{i i} f_{i}\left(p_{i}\right)+\sum_{j \neq i, j=1}^{N} \max \left\{a_{i j} u_{j}, a_{i j} v_{j}\right\} \\
& +\max \left\{\alpha_{i}^{-1} \beta_{i} u_{i} B_{i}, \alpha_{i}^{-1} \beta_{i} v_{i} B_{i}\right\}+I_{i}<0 ;
\end{aligned}
$$

when $x_{i}(t) \in\left(r_{i}, q_{i}\right]$, by virtue of equalities $f_{i}\left(r_{i}\right)=l_{i, 2} r_{i}+c_{i, 2}$ and $f_{i}\left(q_{i}\right)=l_{i, 2} q_{i}+c_{i, 2}$, we get

$$
\begin{aligned}
& \dot{x}_{i}(t)=-d_{i} x_{i}(t)+a_{i i}\left(l_{i, 2} x_{i}(t)+c_{i, 2}\right) \\
& +\sum_{j \neq i, j=1}^{N} a_{i j} f_{j}\left(x_{j}(t)\right)+B_{i} S_{i}(t)+I_{i} \\
& \leq \max \left\{\left(-d_{i}+a_{i i} l_{i, 2}\right) r_{i},\left(-d_{i}+a_{i i} l_{i, 2}\right) q_{i}\right\}+a_{i i} c_{i, 2} \\
& +\sum_{j \neq i, j=1}^{N} a_{i j} f_{j}\left(x_{j}(t)\right)+B_{i} S_{i}(t)+I_{i} \\
& =\max \left\{-d_{i} r_{i}+a_{i i} f_{i}\left(r_{i}\right),-d_{i} q_{i}+a_{i i} f_{i}\left(q_{i}\right)\right\} \\
& +\sum_{j \neq i, j=1}^{N} a_{i j} f_{j}\left(x_{j}(t)\right)+B_{i} S_{i}(t)+I_{i} \\
& \leq \max \left\{-d_{i} r_{i}+a_{i i} f_{i}\left(r_{i}\right),-d_{i} q_{i}+a_{i i} f_{i}\left(q_{i}\right)\right\} \\
& +\sum_{j \neq i, j=1}^{N} \max \left\{a_{i j} u_{j}, a_{i j} v_{j}\right\} \\
& +\max \left\{\alpha_{i}^{-1} \beta_{i} u_{i} B_{i}, \alpha_{i}^{-1} \beta_{i} v_{i} B_{i}\right\}+I_{i} \\
& \leq \max \left\{-d_{i} r_{i}+a_{i i} f_{i}\left(r_{i}\right),-d_{i} p_{i}+a_{i i} f_{i}\left(p_{i}\right)\right\} \\
& +\sum_{j \neq i, j=1}^{N} \max \left\{a_{i j} u_{j}, a_{i j} v_{j}\right\} \\
& +\max \left\{\alpha_{i}^{-1} \beta_{i} u_{i} B_{i}, \alpha_{i}^{-1} \beta_{i} v_{i} B_{i}\right\}+I_{i}<0 ;
\end{aligned}
$$

when $x_{i}(t) \in\left(p_{i}, r_{i}\right]$, it follows from $f_{i}\left(p_{i}\right)=l_{i, 1} p_{i}+$ $c_{i, 1}, f_{i}\left(r_{i}\right)=l_{i, 1} r_{i}+c_{i, 1}$ and system (2) that

$$
\begin{aligned}
\dot{x}_{i}(t)= & -d_{i} x_{i}(t)+a_{i i}\left(l_{i, 1} x_{i}(t)+c_{i, 1}\right) \\
& +\sum_{j \neq i, j=1}^{N} a_{i j} f_{j}\left(x_{j}(t)\right)+B_{i} S_{i}(t)+I_{i} \\
\leq & \max \left\{\left(-d_{i}+a_{i i} l_{i, 1}\right) p_{i},\left(-d_{i}+a_{i i} l_{i, 1}\right) r_{i}\right\}+a_{i i} c_{i, 1}
\end{aligned}
$$




$$
\begin{aligned}
& +\sum_{j \neq i, j=1}^{N} a_{i j} f_{j}\left(x_{j}(t)\right)+B_{i} S_{i}(t)+I_{i} \\
& \leq \max \left\{-d_{i} p_{i}+a_{i i} f_{i}\left(p_{i}\right),-d_{i} r_{i}+a_{i i} f_{i}\left(r_{i}\right)\right\} \\
& +\sum_{j \neq i, j=1}^{N} \max \left\{a_{i j} u_{j}, a_{i j} v_{j}\right\} \\
& +\max \left\{\alpha_{i}^{-1} \beta_{i} u_{i} B_{i}, \alpha_{i}^{-1} \beta_{i} v_{i} B_{i}\right\}+I_{i}<0 .
\end{aligned}
$$

In summary, wherever the initial state $x_{i}(0)\left(i \in \mathbb{N}_{1}\right)$ is located in, $x_{i}(t)$ would flow to and enter the interval $\left(-\infty, p_{i}\right]$ and stay in this interval forever.

Lemma 5. All the state components $x_{i}(t), i \in \mathbb{N}_{3}$, will flow to the interval $\left[r_{i}, q_{i}\right]$ when $t$ tends to $+\infty$.

Proof. We prove it in the following three cases due to the different location of $x_{i}(0)$.

Case (i). Consider $x_{i}(0) \in\left[r_{i}, q_{i}\right]$. In this case, if there exists some $t^{*} \geq 0$ such that $x_{i}\left(t^{*}\right)=r_{i}, r_{i} \leq x_{i}(t) \leq q_{i}$ for $0 \leq t \leq$ $t^{*}$, then we have

$$
\begin{aligned}
\dot{x}_{i}\left(t^{*}\right)= & -d_{i} x_{i}\left(t^{*}\right)+a_{i i} f_{i}\left(x_{i}\left(t^{*}\right)\right) \\
& +\sum_{j \neq i, j=1}^{N} a_{i j} f_{j}\left(x_{j}\left(t^{*}\right)\right)+B_{i} S_{i}\left(t^{*}\right)+I_{i} \\
\geq & -d_{i} r_{i}+a_{i i} f_{i}\left(r_{i}\right)+\sum_{j \neq i, j=1}^{N} \min \left\{a_{i j} u_{j}, a_{i j} v_{j}\right\} \\
& +\min \left\{\alpha_{i}^{-1} \beta_{i} u_{i} B_{i}, \alpha_{i}^{-1} \beta_{i} v_{i} B_{i}\right\}+I_{i}>0 ;
\end{aligned}
$$

similarly, if there exists some $t^{* *} \geq 0$ such that $x_{i}\left(t^{* *}\right)=q_{i}$, $r_{i} \leq x_{i}(t) \leq q_{i}$ for $0 \leq t \leq t^{* *}$, then we get

$$
\begin{aligned}
\dot{x}_{i}\left(t^{* *}\right) \leq & -d_{i} q_{i}+a_{i i} f_{i}\left(q_{i}\right)+\sum_{j \neq i, j=1}^{N} \max \left\{a_{i j} u_{j}, a_{i j} v_{j}\right\} \\
& +\max \left\{\alpha_{i}^{-1} \beta_{i} u_{i} B_{i}, \alpha_{i}^{-1} \beta_{i} v_{i} B_{i}\right\}+I_{i}<0 .
\end{aligned}
$$

From the above two inequalities, we know that if $x_{i}(0) \in$ $\left[r_{i}, q_{i}\right]$, then $x_{i}(t)$ would never get out of this interval. In the same way, we can also obtain that if there exists some $T_{0}>0$ such that $x_{i}\left(T_{0}\right) \in\left[r_{i}, q_{i}\right]$, then $x_{i}(t)$ would stay in it for all $t \geq T_{0}$.

Case (ii). Consider $x_{i}(0) \in\left(-\infty, r_{i}\right)$. When $x_{i}(t) \in\left(-\infty, p_{i}\right]$, from the definition of index subset $\mathbb{N}_{3}$, we get

$$
\begin{aligned}
\dot{x}_{i}(t) \geq & -d_{i} p_{i}+a_{i i} f_{i}\left(p_{i}\right)+\sum_{j \neq i, j=1}^{N} \min \left\{a_{i j} u_{j}, a_{i j} v_{j}\right\} \\
& +\min \left\{\alpha_{i}^{-1} \beta_{i} u_{i} B_{i}, \alpha_{i}^{-1} \beta_{i} v_{i} B_{i}\right\}+I_{i}>0 ;
\end{aligned}
$$

when $x_{i}(t) \in\left(p_{i}, r_{i}\right)$, it follows from the definition of index subset $\mathbb{N}_{3}$, equalities $f_{i}\left(p_{i}\right)=l_{i, 1} p_{i}+c_{i, 1}, f_{i}\left(r_{i}\right)=l_{i, 1} r_{i}+c_{i, 1}$ that

$$
\begin{aligned}
\dot{x}_{i}(t)= & -d_{i} x_{i}(t)+a_{i i}\left(l_{i, 1} x_{i}(t)+c_{i, 1}\right) \\
& +\sum_{j \neq i, j=1}^{N} a_{i j} f_{j}\left(x_{j}(t)\right)+B_{i} S_{i}(t)+I_{i} \\
\geq & \min \left\{\left(-d_{i}+a_{i i} l_{i, 1}\right) p_{i},\left(-d_{i}+a_{i i} l_{i, 1}\right) r_{i}\right\}+a_{i i} c_{i, 1} \\
& +\sum_{j \neq i, j=1}^{N} a_{i j} f_{j}\left(x_{j}(t)\right)+B_{i} S_{i}(t)+I_{i} \\
= & \min \left\{-d_{i} p_{i}+a_{i i} f_{i}\left(p_{i}\right),-d_{i} r_{i}+a_{i i} f_{i}\left(r_{i}\right)\right\} \\
& +\sum_{j \neq i, j=1}^{N} a_{i j} f_{j}\left(x_{j}(t)\right)+B_{i} S_{i}(t)+I_{i} \\
\geq & \min \left\{-d_{i} p_{i}+a_{i i} f_{i}\left(p_{i}\right),-d_{i} r_{i}+a_{i i} f_{i}\left(r_{i}\right)\right\} \\
& +\sum_{j \neq i, j=1}^{N} \min ^{N}\left\{a_{i j} u_{j}, a_{i j} v_{j}\right\} \\
& +\min \left\{\alpha_{i}^{-1} \beta_{i} u_{i} B_{i}, \alpha_{i}^{-1} \beta_{i} v_{i} B_{i}\right\}+I_{i}>0 .
\end{aligned}
$$

Thus, in this case, $x_{i}(t)$ would monotonously increase until it reaches the interval $\left[r_{i}, q_{i}\right]$.

Case (iii). Consider $x_{i}(0) \in\left(q_{i},+\infty\right)$. When $x_{i}(t) \in\left(q_{i},+\infty\right)$, it follows that

$$
\begin{aligned}
\dot{x}_{i}(t) \leq & -d_{i} q_{i}+a_{i i} f_{i}\left(q_{i}\right)+\sum_{j \neq i, j=1}^{N} \max \left\{a_{i j} u_{j}, a_{i j} v_{j}\right\} \\
& +\max \left\{\alpha_{i}^{-1} \beta_{i} u_{i} B_{i}, \alpha_{i}^{-1} \beta_{i} v_{i} B_{i}\right\}+I_{i}<0 .
\end{aligned}
$$

Therefore, $x_{i}(t)$ would monotonously decrease until it enters the interval $\left[r_{i}, q_{i}\right]$.

In summary, wherever the initial state $x_{i}(0)\left(i \in \mathbb{N}_{3}\right)$ is located in, $x_{i}(t)$ would flow to and enter the interval $\left[r_{i}, q_{i}\right]$ and stay in it finally.

Lemma 6. All the state components $x_{i}(t), i \in \mathbb{N}_{4}$, will flow to the interval $\left[q_{i},+\infty\right)$ when $t$ tends to $+\infty$.

Proof. Similar to the proof of Lemmas 4 and 5, we will prove it in the following two cases.

Case (i). Consider $x_{i}(0) \in\left[q_{i},+\infty\right)$. If there exists some $t^{*} \geq 0$ such that $x_{i}\left(t^{*}\right)=q_{i}, x_{i}(t) \geq q_{i}$ for $0 \leq t \leq t^{*}$, then

$$
\begin{aligned}
\dot{x}_{i}\left(t^{*}\right) \geq & -d_{i} q_{i}+a_{i i} f_{i}\left(q_{i}\right)+\sum_{j \neq i, j=1}^{N} \min \left\{a_{i j} u_{j}, a_{i j} v_{j}\right\} \\
& +\min \left\{\alpha_{i}^{-1} \beta_{i} u_{i} B_{i}, \alpha_{i}^{-1} \beta_{i} v_{i} B_{i}\right\}+I_{i}>0 .
\end{aligned}
$$


Therefore, $x_{i}(t)$ would never get out of $\left[q_{i},+\infty\right)$. By the same method, we also get that once $x_{i}\left(T_{0}\right) \in\left[q_{i},+\infty\right)$ for some $T_{0}>0$, then $x_{i}(t)$ would stay in this interval for all $t \geq T_{0}$.

Case (ii). Consider $x_{i}(0) \in\left(-\infty, q_{i}\right)$. In this case, we claim that $x_{i}(t)$ would monotonously increase until it enters the interval $\left[q_{i},+\infty\right)$.

In fact, when $x_{i}(t) \in\left(-\infty, p_{i}\right)$, we have

$$
\begin{aligned}
\dot{x}_{i}(t) \geq & -d_{i} p_{i}+a_{i i} f_{i}\left(p_{i}\right)+\sum_{j \neq i, j=1}^{N} \min \left\{a_{i j} u_{j}, a_{i j} v_{j}\right\} \\
& +\min \left\{\alpha_{i}^{-1} \beta_{i} u_{i} B_{i}, \alpha_{i}^{-1} \beta_{i} v_{i} B_{i}\right\}+I_{i} \\
\geq & -d_{i} q_{i}+a_{i i} f_{i}\left(q_{i}\right)+\sum_{j \neq i, j=1}^{N} \min \left\{a_{i j} u_{j}, a_{i j} v_{j}\right\} \\
& +\min \left\{\alpha_{i}^{-1} \beta_{i} u_{i} B_{i}, \alpha_{i}^{-1} \beta_{i} v_{i} B_{i}\right\}+I_{i}>0 ;
\end{aligned}
$$

when $x_{i}(t) \in\left[p_{i}, r_{i}\right]$, we get

$$
\begin{aligned}
\dot{x}_{i}(t)= & -d_{i} x_{i}(t)+a_{i i}\left(l_{i, 1} x_{i}(t)+c_{i, 1}\right) \\
& +\sum_{j \neq i, j=1}^{N} a_{i j} f_{j}\left(x_{j}(t)\right)+B_{i} S_{i}(t)+I_{i} \\
\geq & \min \left\{-d_{i} p_{i}+a_{i i} f_{i}\left(p_{i}\right),-d_{i} r_{i}+a_{i i} f_{i}\left(r_{i}\right)\right\} \\
& +\sum_{j \neq i, j=1}^{N} a_{i j} f_{j}\left(x_{j}(t)\right)+B_{i} S_{i}(t)+I_{i} \\
\geq & \min \left\{-d_{i} q_{i}+a_{i i} f_{i}\left(q_{i}\right),-d_{i} r_{i}+a_{i i} f_{i}\left(r_{i}\right)\right\} \\
& +\sum_{j \neq i, j=1}^{N} \min \left\{a_{i j} u_{j}, a_{i j} v_{j}\right\} \\
& +\min \left\{\alpha_{i}^{-1} \beta_{i} u_{i} B_{i}, \alpha_{i}^{-1} \beta_{i} v_{i} B_{i}\right\}+I_{i}>0 ;
\end{aligned}
$$

when $x_{i}(t) \in\left(r_{i}, q_{i}\right)$, then we obtain

$$
\begin{aligned}
\dot{x}_{i}(t)= & -d_{i} x_{i}(t)+a_{i i}\left(l_{i, 2} x_{i}(t)+c_{i, 2}\right) \\
& +\sum_{j \neq i, j=1}^{N} a_{i j} f_{j}\left(x_{j}(t)\right)+B_{i} S_{i}(t)+I_{i} \\
\geq & \min \left\{-d_{i} q_{i}+a_{i i} f_{i}\left(q_{i}\right),-d_{i} r_{i}+a_{i i} f_{i}\left(r_{i}\right)\right\} \\
& +\sum_{j \neq i, j=1}^{N} \min \left\{a_{i j} u_{j}, a_{i j} v_{j}\right\} \\
& +\min \left\{\alpha_{i}^{-1} \beta_{i} u_{i} B_{i}, \alpha_{i}^{-1} \beta_{i} v_{i} B_{i}\right\}+I_{i}>0 .
\end{aligned}
$$

In summary, wherever the initial state $x_{i}(0)\left(i \in \mathbb{N}_{4}\right)$ is located in, $x_{i}(t)$ would flow to $\left[q_{i},+\infty\right)$ when $t$ is big enough and stay in it forever.
Denote $\left(-\infty, p_{i}\right]=\left(-\infty, p_{i}\right]^{1} \times\left[p_{i}, r_{i}\right]^{0} \times\left[r_{i},+\infty\right)^{0}$; $\left[p_{i}, r_{i}\right]=\left(-\infty, p_{i}\right]^{0} \times\left[p_{i}, r_{i}\right]^{1} \times\left[r_{i},+\infty\right)^{0} ;\left[r_{i},+\infty\right)=$ $\left(-\infty, p_{i}\right]^{0} \times\left[p_{i}, r_{i}\right]^{0} \times\left[r_{i},+\infty\right)^{1}$. For any $i \in \mathbb{N}_{2}$, let $\delta_{i}=$ $\left(\delta_{i}^{(1)}, \delta_{i}^{(2)}, \delta_{i}^{(3)}\right)=(1,0,0)$ or $(0,1,0)$ or $(0,0,1)$ and define

$$
\begin{aligned}
\Omega_{\delta_{i}} & =\left(-\infty, p_{i}\right]_{i}^{\delta_{i}^{(1)}} \times\left[p_{i}, r_{i}\right]^{\delta_{i}^{(2)}} \times\left[r_{i},+\infty\right)^{\delta_{i}^{(3)}} \\
\Omega_{\delta} & =\prod_{i \in \mathbb{N}_{1}}\left(-\infty, p_{i}\right] \times \prod_{i \in \mathbb{N}_{2}} \Omega_{\delta_{i}} \times \prod_{i \in \mathbb{N}_{3}}\left[r_{i}, q_{i}\right] \times \prod_{i \in \mathbb{N}_{4}}\left[q_{i},+\infty\right) .
\end{aligned}
$$

It is easy to see that there exist $3^{\sharp \mathbb{N}_{2}} \Omega_{\delta}$-type regions, where $\sharp \mathbb{N}_{2}$ denotes the number of elements in the set $\mathbb{N}_{2}$. Now, we will prove the following theorem on the existence of multiple equilibria for system (2).

Theorem 7. Suppose that $\mathbb{N}_{1} \cup \mathbb{N}_{2} \cup \mathbb{N}_{3} \cup \mathbb{N}_{4}=\{1,2, \ldots, N\}$. Then, system (2) with activation functions (3) has $3^{\sharp \mathbb{N}_{2}}$ equilibria.

Proof. Pick a region arbitrarily $\Omega_{\delta} \times \prod_{i=1}^{N}\left[\alpha_{i}^{-1} \beta_{i} u_{i}, \alpha_{i}^{-1} \beta_{i} v_{i}\right]$; we will show that there exists an equilibrium point located in each $\Omega_{\delta} \times \prod_{i=1}^{N}\left[\alpha_{i}^{-1} \beta_{i} u_{i}, \alpha_{i}^{-1} \beta_{i} v_{i}\right]$.

Denote $\mathbb{N}_{2,1}=\left\{i \in \mathbb{N}_{2}: \delta_{i}^{(1)}=1\right\}, \mathbb{N}_{2,2}=\left\{i \in \mathbb{N}_{2}\right.$ : $\left.\delta_{i}^{(2)}=1\right\}$, and $\mathbb{N}_{2,3}=\left\{i \in \mathbb{N}_{2}: \delta_{i}^{(3)}=1\right\}$. It is easy to see that $\mathbb{N}_{2,1} \cup \mathbb{N}_{2,2} \cup \mathbb{N}_{2,3}=\mathbb{N}_{2}$.

Let $\left(x^{T}(t), S^{T}(t)\right)^{T}$ be any solution of system (2) with initial state $\left(x^{T}(0), S^{T}(0)\right)^{T} \in \Omega_{\delta} \times \prod_{i=1}^{N}\left[\alpha_{i}^{-1} \beta_{i} u_{i}, \alpha_{i}^{-1} \beta_{i} v_{i}\right]$. Then, for $i \in \mathbb{N}_{2,1}$, if there exists some $t^{*} \geq 0$ such that $x_{i}\left(t^{*}\right)=p_{i}$, then we get from the definition of index subset $\mathbb{N}_{2}$ that

$$
\begin{aligned}
\dot{x}_{i}\left(t^{*}\right)= & -d_{i} x_{i}\left(t^{*}\right)+a_{i i} f_{i}\left(x_{i}\left(t^{*}\right)\right) \\
& +\sum_{j \neq i, j=1}^{N} a_{i j} f_{j}\left(x_{j}\left(t^{*}\right)\right)+B_{i} S_{i}\left(t^{*}\right)+I_{i} \\
\leq & -d_{i} p_{i}+a_{i i} f_{i}\left(p_{i}\right)+\sum_{j \neq i, j=1}^{N} \max \left\{a_{i j} u_{j}, a_{i j} v_{j}\right\} \\
& +\max \left\{\alpha_{i}^{-1} \beta_{i} u_{i} B_{i}, \alpha_{i}^{-1} \beta_{i} v_{i} B_{i}\right\}+I_{i}<0 .
\end{aligned}
$$

Similarly, for $i \in \mathbb{N}_{2,3}$, if $x_{i}(t) \geq q_{i}$, note that $f_{i}\left(p_{i}\right)=f_{i}\left(q_{i}\right)$; then

$$
\begin{aligned}
\dot{x}_{i}(t) \leq & -d_{i} q_{i}+a_{i i} f_{i}\left(q_{i}\right)+\sum_{j \neq i, j=1}^{N} \max \left\{a_{i j} u_{j}, a_{i j} v_{j}\right\} \\
& +\max \left\{\alpha_{i}^{-1} \beta_{i} u_{i} B_{i}, \alpha_{i}^{-1} \beta_{i} v_{i} B_{i}\right\}+I_{i} \\
\leq & -d_{i} p_{i}+a_{i i} f_{i}\left(p_{i}\right)+\sum_{j \neq i, j=1}^{N} \max \left\{a_{i j} u_{j}, a_{i j} v_{j}\right\} \\
& +\max \left\{\alpha_{i}^{-1} \beta_{i} u_{i} B_{i}, \alpha_{i}^{-1} \beta_{i} v_{i} B_{i}\right\}+I_{i}<0,
\end{aligned}
$$


and if there exists some $t^{* *} \geq 0$ such that $x_{i}\left(t^{* *}\right)=r_{i}$, then

$$
\begin{aligned}
\dot{x}_{i}\left(t^{* *}\right) \geq & -d_{i} r_{i}+a_{i i} f_{i}\left(r_{i}\right)+\sum_{j \neq i, j=1}^{N} \min \left\{a_{i j} u_{j}, a_{i j} v_{j}\right\} \\
& +\min \left\{\alpha_{i}^{-1} \beta_{i} u_{i} B_{i}, \alpha_{i}^{-1} \beta_{i} v_{i} B_{i}\right\}+I_{i}>0 .
\end{aligned}
$$

That is, the trajectory $x_{i}(t)$ with $x_{i}(0) \in\left[r_{i},+\infty\right), i \in \mathbb{N}_{2,3}$, would enter and stay in the interval $\left[r_{i}, q_{i}\right]$, which implies that there does not exist any equilibria with the corresponding $i$ th state component located in $\left[q_{i},+\infty\right)$.

Combining with Lemmas $4-6$, it can be concluded that $x_{i}(t), i \notin \mathbb{N}_{2,2}$, would never escape from the corresponding interval of $\Omega_{\delta}$. Furthermore, denote

$$
\begin{aligned}
\widetilde{\Omega}_{\delta}= & \prod_{i \in \mathbb{N}_{1} \cup \mathbb{N}_{2,1}}\left(-\infty, p_{i}\right] \times \prod_{i \in \mathbb{N}_{2,2}}\left[p_{i}, r_{i}\right] \\
& \times \prod_{i \in \mathbb{N}_{2,3} \cup \mathbb{N}_{3}}\left[r_{i}, q_{i}\right] \times \prod_{i \in \mathbb{N}_{4}}\left[q_{i},+\infty\right) .
\end{aligned}
$$

Then, from (26)-(28), we can derive that if $\Omega_{\delta} \times \prod_{i=1}^{N}\left[\alpha_{i}^{-1} \beta_{i} u_{i}, \alpha_{i}^{-1} \beta_{i} v_{i}\right]$ has an equilibrium point, it must be located in $\widetilde{\Omega}_{\delta} \times \prod_{i=1}^{N}\left[\alpha_{i}^{-1} \beta_{i} u_{i}, \alpha_{i}^{-1} \beta_{i} v_{i}\right]$.

Note that any equilibrium point of system (2) is a root of the following equations:

$$
\begin{aligned}
& -d_{i} x_{i}+\sum_{j=1}^{N} a_{i j} f_{j}\left(x_{j}\right)+B_{i} S_{i}+I_{i}=0, \\
& -\alpha_{i} S_{i}+\beta_{i} f_{i}\left(x_{i}\right)=0, \quad i=1,2, \ldots, N .
\end{aligned}
$$

Equivalently, the above equations can be rewritten as

$$
\begin{gathered}
-d_{i} x_{i}+\left(a_{i i}+\alpha_{i}^{-1} B_{i} \beta_{i}\right) f_{i}\left(x_{i}\right)+\sum_{j \neq i, j=1}^{N} a_{i j} f_{j}\left(x_{j}\right)+I_{i}=0, \\
S_{i}=\alpha_{i}^{-1} \beta_{i} f_{i}\left(x_{i}\right), \quad i=1,2, \ldots, N
\end{gathered}
$$

In $\widetilde{\Omega}_{\delta} \times \prod_{i=1}^{N}\left[\alpha_{i}^{-1} \beta_{i} u_{i}, \alpha_{i}^{-1} \beta_{i} v_{i}\right]$, any equilibrium point of system (2) satisfies the following equations:

$$
\begin{aligned}
& -d_{i} x_{i}+\left(a_{i i}+\alpha_{i}^{-1} \beta_{i} B_{i}\right) u_{i}+\sum_{\substack{j \in \mathbb{N}_{1} \cup \mathbb{N}_{2,1} \\
j \neq i}} a_{i j} u_{j} \\
& +\sum_{j \in \mathbb{N}_{2,2}} a_{i j}\left(l_{j, 1} x_{j}+c_{j, 1}\right)+\sum_{j \in \mathbb{N}_{2,3} \cup \mathbb{N}_{3}} a_{i j}\left(l_{j, 2} x_{j}+c_{j, 2}\right) \\
& +\sum_{j \in \mathbb{N}_{4}} a_{i j} u_{j}+I_{i}=0, \quad i \in \mathbb{N}_{1} \cup \mathbb{N}_{2,1}, \\
& -d_{i} x_{i}+\left(a_{i i}+\alpha_{i}^{-1} \beta_{i} B_{i}\right)\left(l_{i, 1} x_{i}+c_{i, 1}\right)+\sum_{j \in \mathbb{N}_{1} \cup \mathbb{N}_{2,1}} a_{i j} u_{j} \\
& +\sum_{\substack{j \in \mathbb{N}_{2,2} \\
j \neq i}} a_{i j}\left(l_{j, 1} x_{j}+c_{j, 1}\right)+\sum_{j \in \mathbb{N}_{2,3} \cup \mathbb{N}_{3}} a_{i j}\left(l_{j, 2} x_{j}+c_{j, 2}\right) \\
& +\sum_{j \in \mathbb{N}_{4}} a_{i j} u_{j}+I_{i}=0, \quad i \in \mathbb{N}_{2,2} \\
& -d_{i} x_{i}+\left(a_{i i}+\alpha_{i}^{-1} \beta_{i} B_{i}\right)\left(l_{i, 2} x_{i}+c_{i, 2}\right)+\sum_{j \in \mathbb{N}_{1} \cup \mathbb{N}_{2,1}} a_{i j} u_{j} \\
& +\sum_{j \in \mathbb{N}_{2,2}} a_{i j}\left(l_{j, 1} x_{j}+c_{j, 1}\right)+\sum_{\substack{j \in \mathbb{N}_{2,3} \cup \mathbb{N}_{3} \\
j \neq i}} a_{i j}\left(l_{j, 2} x_{j}+c_{j, 2}\right) \\
& +\sum_{j \in \mathbb{N}_{4}} a_{i j} u_{j}+I_{i}=0, \quad i \in \mathbb{N}_{2,3} \cup \mathbb{N}_{3}, \\
& -d_{i} x_{i}+\left(a_{i i}+\alpha_{i}^{-1} \beta_{i} B_{i}\right) u_{i}+\sum_{j \in \mathbb{N}_{1} \cup \mathbb{N}_{2,1}} a_{i j} u_{j} \\
& +\sum_{j \in \mathbb{N}_{2,2}} a_{i j}\left(l_{j, 1} x_{j}+c_{j, 1}\right)+\sum_{j \in \mathbb{N}_{2,3} \cup \mathbb{N}_{3}} a_{i j}\left(l_{j, 2} x_{j}+c_{j, 2}\right) \\
& +\sum_{\substack{j \in \mathbb{N}_{4} \\
j \neq i}} a_{i j} u_{j}+I_{i}=0, \quad i \in \mathbb{N}_{4} .
\end{aligned}
$$

In the subset region $\prod_{i \in \mathbb{N}_{2,2}}\left[p_{i}, r_{i}\right] \times \prod_{i \in \mathbb{N}_{2,3} \cup \mathbb{N}_{3}}\left[r_{i}, q_{i}\right]$, define a map $\Gamma$ as follows:

$$
\begin{aligned}
& \Gamma_{i}\left(x_{i}\right)=\frac{1}{d_{i}-\left(a_{i i}+\alpha_{i}^{-1} \beta_{i} B_{i}\right) l_{i, 1}} \\
& \times\left[\left(a_{i i}+\alpha_{i}^{-1} \beta_{i} B_{i}\right) c_{i, 1}+\sum_{j \in \mathbb{N}_{1} \cup \mathbb{N}_{2,1}} a_{i j} u_{j}\right. \\
& +\sum_{\substack{j \in \mathbb{N}_{2,2} \\
j \neq i}} a_{i j}\left(l_{j, 1} x_{j}+c_{j, 1}\right) \\
& +\sum_{j \in \mathbb{N}_{2,3} \cup \mathbb{N}_{3}} a_{i j}\left(l_{j, 2} x_{j}+c_{j, 2}\right) \\
& \left.+\sum_{j \in \mathbb{N}_{4}} a_{i j} u_{j}+I_{i}\right], \quad i \in \mathbb{N}_{2,2},
\end{aligned}
$$




$$
\begin{aligned}
& \Gamma_{i}\left(x_{i}\right)=\frac{1}{d_{i}-\left(a_{i i}+\alpha_{i}^{-1} \beta_{i} B_{i}\right) l_{i, 2}} \\
& \times\left[\left(a_{i i}+\alpha_{i}^{-1} \beta_{i} B_{i}\right) c_{i, 2}+\sum_{j \in \mathbb{N}_{1} \cup \mathbb{N}_{2,1}} a_{i j} u_{j}\right. \\
& +\sum_{j \in \mathbb{N}_{2,2}} a_{i j}\left(l_{j, 1} x_{j}+c_{j, 1}\right) \\
& +\sum_{\substack{j \in \mathbb{N}_{2,3} \cup \mathbb{N}_{3} \\
j \neq i}} a_{i j}\left(l_{j, 2} x_{j}+c_{j, 2}\right) \\
& \left.+\sum_{j \in \mathbb{N}_{4}} a_{i j} u_{j}+I_{i}\right], \quad i \in \mathbb{N}_{2,3} \cup \mathbb{N}_{3} .
\end{aligned}
$$

For $i \in \mathbb{N}_{2,2}$, substituting $f_{i}\left(p_{i}\right)=l_{i, 1} p_{i}+c_{i, 1}$ into (6) and noting that $d_{i}-\left(a_{i i}+\alpha_{i}^{-1} \beta_{i} B_{i}\right) l_{i, 1}<0$ (Remark 2$)$, we get that $\Gamma_{i}\left(x_{i}\right)$

$$
\begin{aligned}
& \geq \frac{1}{d_{i}-\left(a_{i i}+\alpha_{i}^{-1} \beta_{i} B_{i}\right) l_{i, 1}} \\
& \quad \times\left[\left(a_{i i}+\alpha_{i}^{-1} \beta_{i} B_{i}\right) c_{i, 1}+\sum_{j \neq i, j=1}^{N} \max \left\{a_{i j} u_{j}, a_{i j} v_{j}\right\}+I_{i}\right] \\
& >p_{i} .
\end{aligned}
$$

Similarly, substituting $f_{i}\left(r_{i}\right)=l_{i, 1} r_{i}+c_{i, 1}$ into (7) results in

$$
\begin{aligned}
\Gamma_{i}\left(x_{i}\right) & \\
\leq & \frac{1}{d_{i}-\left(a_{i i}+\alpha_{i}^{-1} \beta_{i} B_{i}\right) l_{i, 1}} \\
& \times\left[\left(a_{i i}+\alpha_{i}^{-1} \beta_{i} B_{i}\right) c_{i, 1}+\sum_{j \neq i, j=1}^{N} \min \left\{a_{i j} u_{j}, a_{i j} v_{j}\right\}+I_{i}\right] \\
& <r_{i} .
\end{aligned}
$$

For $i \in \mathbb{N}_{2,3}$, note that $-d_{i} p_{i}>-d_{i} q_{i}, f_{i}\left(p_{i}\right)=f_{i}\left(q_{i}\right)=l_{i, 2} q_{i}+$ $c_{i, 2}$, and $f_{i}\left(r_{i}\right)=l_{i, 2} r_{i}+c_{i, 2}$; it follows from (6)-(7) and $d_{i}-$ $\left(a_{i i}+\alpha_{i}^{-1} \beta_{i} B_{i}\right) l_{i, 2}>0($ Remark 3$)$ that

$\Gamma_{i}\left(x_{i}\right)$

$$
\begin{aligned}
\geq & \frac{1}{d_{i}-\left(a_{i i}+\alpha_{i}^{-1} \beta_{i} B_{i}\right) l_{i, 2}} \\
& \times\left[\left(a_{i i}+\alpha_{i}^{-1} \beta_{i} B_{i}\right) c_{i, 2}+\sum_{j \neq i, j=1}^{N} \min \left\{a_{i j} u_{j}, a_{i j} v_{j}\right\}+I_{i}\right]
\end{aligned}
$$$$
>r_{i}
$$

$$
\begin{aligned}
\Gamma_{i}\left(x_{i}\right) & \\
\leq & \frac{1}{d_{i}-\left(a_{i i}+\alpha_{i}^{-1} \beta_{i} B_{i}\right) l_{i, 2}} \\
& \times\left[\left(a_{i i}+\alpha_{i}^{-1} \beta_{i} B_{i}\right) c_{i, 2}+\sum_{j \neq i, j=1}^{N} \max \left\{a_{i j} u_{j}, a_{i j} v_{j}\right\}+I_{i}\right]
\end{aligned}
$$$$
<q_{i}
$$

For $i \in \mathbb{N}_{3}$, note that $v_{i}=f_{i}\left(r_{i}\right)=l_{i, 2} r_{i}+c_{i, 2}$ and $u_{i}=f_{i}\left(q_{i}\right)=$ $l_{i, 2} q_{i}+c_{i, 2}$; we can get from the definition of index subset $\mathbb{N}_{3}$ that

$$
\begin{aligned}
& -d_{i} r_{i}+\left(a_{i i}+\alpha_{i}^{-1} \beta_{i} B_{i}\right)\left(l_{i, 2} r_{i}+c_{i, 2}\right) \\
& +\sum_{j \neq i, j=1}^{N} \min \left\{a_{i j} u_{j}, a_{i j} v_{j}\right\}+I_{i}>0, \\
& -d_{i} q_{i}+\left(a_{i i}+\alpha_{i}^{-1} \beta_{i} B_{i}\right)\left(l_{i, 2} q_{i}+c_{i, 2}\right) \\
& +\sum_{j \neq i, j=1}^{N} \max \left\{a_{i j} u_{j}, a_{i j} v_{j}\right\}+I_{i}<0 .
\end{aligned}
$$

That is,

$$
\begin{aligned}
& {\left[-d_{i}+\left(a_{i i}+\alpha_{i}^{-1} \beta_{i} B_{i}\right) l_{i, 2}\right] r_{i}+\left(a_{i i}+\alpha_{i}^{-1} \beta_{i} B_{i}\right) c_{i, 2}} \\
& +\sum_{j \neq i, j=1}^{N} \min \left\{a_{i j} u_{j}, a_{i j} v_{j}\right\}+I_{i}>0, \\
& {\left[-d_{i}+\left(a_{i i}+\alpha_{i}^{-1} \beta_{i} B_{i}\right) l_{i, 2}\right] q_{i}+\left(a_{i i}+\alpha_{i}^{-1} \beta_{i} B_{i}\right) c_{i, 2}} \\
& +\sum_{j \neq i, j=1}^{N} \max \left\{a_{i j} u_{j}, a_{i j} v_{j}\right\}+I_{i}<0 .
\end{aligned}
$$

Then combining inequality $d_{i}-\left(a_{i i}+\alpha_{i}^{-1} \beta_{i} B_{i}\right) l_{i, 2}>0$ and (38) together gives

$$
\begin{aligned}
& \Gamma_{i}\left(x_{i}\right) \\
& \quad \geq \frac{1}{d_{i}-\left(a_{i i}+\alpha_{i}^{-1} \beta_{i} B_{i}\right) l_{i, 2}} \\
& \quad \times\left[\left(a_{i i}+\alpha_{i}^{-1} \beta_{i} B_{i}\right) c_{i, 2}+\sum_{j \neq i, j=1}^{N} \min \left\{a_{i j} u_{j}, a_{i j} v_{j}\right\}+I_{i}\right] \\
& \quad>r_{i},
\end{aligned}
$$




$$
\begin{aligned}
& \Gamma_{i}\left(x_{i}\right) \\
& \leq \leq \frac{1}{d_{i}-\left(a_{i i}+\alpha_{i}^{-1} \beta_{i} B_{i}\right) l_{i, 2}} \\
& \quad \times\left[\left(a_{i i}+\alpha_{i}^{-1} \beta_{i} B_{i}\right) c_{i, 2}+\sum_{j \neq i, j=1}^{N} \max \left\{a_{i j} u_{j}, a_{i j} v_{j}\right\}+I_{i}\right] \\
& \quad<q_{i} .
\end{aligned}
$$

Therefore, the map $\Gamma$ maps a bounded and closed set into itself. Applying Brouwer's fixed point theorem, there exists one $\left(x_{i_{1}}^{*}, x_{i_{2}}^{*}, \ldots, x_{i_{s}}^{*}\right)^{T} \in \prod_{i \in \mathbb{N}_{2,2}}\left[p_{i}, r_{i}\right] \times \prod_{i \in \mathbb{N}_{2,3} \cup \mathbb{N}_{3}}\left[r_{i}, q_{i}\right]$ such that

$$
\Gamma\left(x_{i_{1}}^{*}, x_{i_{2}}^{*}, \ldots, x_{i_{s}}^{*}\right)=\left(x_{i_{1}}^{*}, x_{i_{2}}^{*}, \ldots, x_{i_{s}}^{*}\right),
$$

where $i_{1}, i_{2}, \ldots, i_{s}$ represent the elements of index subset $\mathbb{N}_{2,2} \cup \mathbb{N}_{2,3} \cup \mathbb{N}_{3}$.

Then for $i \in \mathbb{N}_{1} \cup \mathbb{N}_{2,1}$, define

$$
\begin{aligned}
F_{i}\left(x_{i}\right)= & -d_{i} x_{i}+\left(a_{i i}+\alpha_{i}^{-1} \beta_{i} B_{i}\right) u_{i} \\
& +\sum_{\substack{j \in \mathbb{N}_{1} \cup \mathbb{N}_{2,1} \\
j \neq i}} a_{i j} u_{j}+\sum_{j \in \mathbb{N}_{2,2}} a_{i j}\left(l_{j, 1} x_{j}^{*}+c_{j, 1}\right) \\
& +\sum_{j \in \mathbb{N}_{2,3} \cup \mathbb{N}_{3}} a_{i j}\left(l_{j, 2} x_{j}^{*}+c_{j, 2}\right)+\sum_{j \in \mathbb{N}_{4}} a_{i j} u_{j}+I_{i} .
\end{aligned}
$$

By virtue of the definition of index subsets $\mathbb{N}_{1}$ and $\mathbb{N}_{2}$, we have $\lim _{\xi \rightarrow-\infty} F_{i}(\xi)=+\infty$ and

$$
\begin{aligned}
F_{i}\left(p_{i}\right) \leq & -d_{i} p_{i}+\left(a_{i i}+\alpha_{i}^{-1} \beta_{i} B_{i}\right) u_{i} \\
& +\sum_{j \neq i, j=1}^{N} \max \left\{a_{i j} u_{j}, a_{i j} v_{j}\right\}+I_{i}<0 .
\end{aligned}
$$

Therefore, there exists the unique $x_{i}^{*} \in\left(-\infty, p_{i}\right)$ such that $F_{i}\left(x_{i}^{*}\right)=0$.

Similarly, for $i \in \mathbb{N}_{4}$, define

$$
\begin{aligned}
F_{i}\left(x_{i}\right)= & -d_{i} x_{i}+\left(a_{i i}+\alpha_{i}^{-1} \beta_{i} B_{i}\right) u_{i} \\
& +\sum_{j \in \mathbb{N}_{1} \cup \mathbb{N}_{2,1}} a_{i j} u_{j}+\sum_{j \in \mathbb{N}_{2,2}} a_{i j}\left(l_{j, 1} x_{j}^{*}+c_{j, 1}\right) \\
& +\sum_{j \in \mathbb{N}_{2,3} \cup \mathbb{N}_{3}} a_{i j}\left(l_{j, 2} x_{j}^{*}+c_{j, 2}\right)+\sum_{\substack{j \in \mathbb{N}_{4} \\
j \neq i}} a_{i j} u_{j}+I_{i},
\end{aligned}
$$

and we can also derive that there exists the unique $x_{i}^{*} \epsilon$ $\left(q_{i},+\infty\right)$ such that $F_{i}\left(x_{i}^{*}\right)=0$.

Denote $\left(x^{* T}, S^{* T}\right)^{T}=\left(x_{1}^{*}, x_{2}^{*}, \ldots, x_{N}^{*}, \beta_{1} \alpha_{1}^{-1} f_{1}\left(x_{1}^{*}\right)\right.$, $\left.\beta_{2} \alpha_{2}^{-1} f_{2}\left(x_{2}^{*}\right), \ldots, \beta_{N} \alpha_{N}^{-1} f_{N}\left(x_{N}^{*}\right)\right)^{T}$. It is easy to see that $\left(x^{* T}, S^{* T}\right)^{T}$ is the equilibrium point located in subset $\widetilde{\Omega}_{\delta} \times$ $\prod_{i=1}^{N}\left[\alpha_{i}^{-1} \beta_{i} u_{i}, \alpha_{i}^{-1} \beta_{i} v_{i}\right]$, which is also the equilibrium point located in subset $\Omega_{\delta} \times \prod_{i=1}^{N}\left[\alpha_{i}^{-1} \beta_{i} u_{i}, \alpha_{i}^{-1} \beta_{i} v_{i}\right]$. It should be noted that, by the definition of $\mathbb{N}_{1}-\mathbb{N}_{4}$, any state component of $x^{*}$ cannot touch the boundary of $\Omega_{\delta}$. That is, $x^{*}$ is located in the interior of $\Omega_{\delta}$ (see Remark 8). Therefore, system (2) has $3^{\sharp \mathbb{N}_{2}}$ equilibria.

Remark 8. $x^{*}$ is located in the interior of $\Omega_{\delta}$.

Proof. Note that $x^{*}$ satisfies the following equations:

$$
\begin{aligned}
-d_{i} x_{i}^{*} & +\left(a_{i i}+\alpha_{i}^{-1} B_{i} \beta_{i}\right) f_{i}\left(x_{i}^{*}\right) \\
& +\sum_{j \neq i, j=1}^{N} a_{i j} f_{j}\left(x_{j}^{*}\right)+I_{i}=0, \quad i=1,2, \ldots, N .
\end{aligned}
$$

In the following, we prove that $x_{i}^{*} \neq p_{i}, i \in \mathbb{N}_{1}$. Otherwise, from the definition of $\mathbb{N}_{1}$, we have

$$
\begin{aligned}
0= & -d_{i} p_{i}+\left(a_{i i}+\alpha_{i}^{-1} B_{i} \beta_{i}\right) f_{i}\left(p_{i}\right) \\
& +\sum_{j \neq i, j=1}^{N} a_{i j} f_{j}\left(x_{j}^{*}\right)+I_{i} \\
\leq & -d_{i} p_{i}+a_{i i} f_{i}\left(p_{i}\right)+\sum_{j \neq i, j=1}^{N} \max \left\{a_{i j} u_{j} a_{i j} v_{j}\right\} \\
& +\max \left\{\alpha_{i}^{-1} \beta_{i} u_{i} B_{i}, \alpha_{i}^{-1} \beta_{i} v_{i} B_{i}\right\}+I_{i}<0,
\end{aligned}
$$

that is a contradiction. Similarly, from the definition of $\mathbb{N}_{2}-\mathbb{N}_{4}$, we can also get

$$
\begin{aligned}
& x_{i}^{*} \neq p_{i}, r_{i}, q_{i}, \quad i \in \mathbb{N}_{2}, \\
& x_{i}^{*} \neq r_{i}, q_{i}, \quad i \in \mathbb{N}_{3}, \\
& x_{i}^{*} \neq q_{i}, \quad i \in \mathbb{N}_{4} .
\end{aligned}
$$

That is, $x^{*}$ is located in the interior of $\Omega_{\delta}$.

Remark 9. Suppose that $\mathbb{N}_{1} \cup \mathbb{N}_{2} \cup \mathbb{N}_{3} \cup \mathbb{N}_{4}=\{1,2, \ldots, N\}$. Furthermore, if the following conditions

$$
\begin{aligned}
& \sum_{\substack{j \in \mathbb{N}_{2,2} \\
j \neq i}}\left|a_{i j} l_{j, 1}\right|+\sum_{j \in \mathbb{N}_{2,3} \cup \mathbb{N}_{3}}\left|a_{i j} l_{j, 2}\right| \\
& <\left(a_{i i}+\alpha_{i}^{-1} B_{i} \beta_{i}\right) l_{i, 1}-d_{i}, \quad i \in \mathbb{N}_{2,2}, \\
& \sum_{j \in \mathbb{N}_{2,2}}\left|a_{i j} l_{j, 1}\right|+\sum_{\substack{j \in \mathbb{N}_{2,3} \cup \mathbb{N}_{3} \\
j \neq i}}\left|a_{i j} l_{j, 2}\right| \\
& <d_{i}-\left(a_{i i}+\alpha_{i}^{-1} B_{i} \beta_{i}\right) l_{i, 2}, \quad i \in \mathbb{N}_{2,3} \cup \mathbb{N}_{3},
\end{aligned}
$$

hold, then system (2) with activation functions (3) can have and only have $3^{\sharp \mathbb{N}_{2}}$ equilibria.

Proof. From the proof of Theorem 7, we only need to prove that the fixed point of $\Gamma$ is unique. In fact, suppose that 
there exists another fixed point $\left(x_{i_{1}}^{* *}, x_{i_{2}}^{* *}, \ldots, x_{i_{s}}^{* *}\right)^{T} \in$ $\prod_{i \in \mathbb{N}_{2,2}}\left[p_{i}, r_{i}\right] \times \prod_{i \in \mathbb{N}_{2,3} \cup \mathbb{N}_{3}}\left[r_{i}, q_{i}\right]$ such that

$$
\Gamma\left(x_{i_{1}}^{* *}, x_{i_{2}}^{* *}, \ldots, x_{i_{s}}^{* *}\right)=\left(x_{i_{1}}^{* *}, x_{i_{2}}^{* *}, \ldots, x_{i_{s}}^{* *}\right) .
$$

Denote the index $i_{l}$ such that

$$
\left|x_{i_{l}}^{*}-x_{i_{l}}^{* *}\right|=\max _{i \in \mathbb{N}_{2,2} \cup \mathbb{N}_{2,3} \cup \mathbb{N}_{3}}\left|x_{i}^{*}-x_{i}^{* *}\right| \text {. }
$$

If $i_{l} \in \mathbb{N}_{2,2}$, from $\left(a_{i_{l} i_{l}}+\alpha_{i_{l}}^{-1} \beta_{i_{l}} B_{i_{l}}\right) l_{i_{l}, 1}-d_{i_{l}}>0$ (Remark 2) and (47), we have

$$
\begin{aligned}
\left|x_{i_{l}}^{*}-x_{i_{l}}^{* *}\right|= & \left|\Gamma_{i_{l}}\left(x_{i_{l}}^{*}\right)-\Gamma_{i_{l}}\left(x_{i_{l}}^{* *}\right)\right| \\
= & \frac{1}{\left(a_{i_{l} i_{l}}+\alpha_{i_{l}}^{-1} \beta_{i_{l}} B_{i_{l}}\right) l_{i_{l}, 1}-d_{i_{l}}} \\
& \times \mid \sum_{\substack{j \in \mathbb{N}_{2,2} \\
j \neq i}} a_{i_{l} j} l_{j, 1}\left(x_{j}^{*}-x_{j}^{* *}\right) \\
& +\sum_{j \in \mathbb{N}_{2,3} \cup \mathbb{N}_{3}} a_{i_{l} j} l_{j, 2}\left(x_{j}^{*}-x_{j}^{* *}\right) \mid \\
\leq & \frac{\sum_{j \in \mathbb{N}_{2,2}, j \neq i_{l}}\left|a_{i_{l} j} l_{j, 1}\right|+\sum_{j \in \mathbb{N}_{2,3} \cup \mathbb{N}_{3}}\left|a_{i_{l} j} l_{j, 2}\right|}{\left(a_{i_{i} i_{l}}+\alpha_{i_{l}}^{-1} \beta_{i_{l}} B_{i_{l}}\right) l_{i_{l}, 1}-d_{i_{l}}} \\
& \times\left|x_{i_{l}}^{*}-x_{i_{l}}^{* *}\right|<\left|x_{i_{l}}^{*}-x_{i_{l}}^{* *}\right| .
\end{aligned}
$$

If $i_{l} \in \mathbb{N}_{2,3} \cup \mathbb{N}_{3}$, from $d_{i_{l}}-\left(a_{i_{l} i_{l}}+\alpha_{i_{l}}^{-1} \beta_{i_{l}} B_{i_{l}}\right) l_{i_{l}, 2}>0$ (Remark 3$)$ and (48), we have

$$
\begin{aligned}
\left|x_{i_{l}}^{*}-x_{i_{l}}^{* *}\right|= & \left|\Gamma_{i_{l}}\left(x_{i_{l}}^{*}\right)-\Gamma_{i_{l}}\left(x_{i_{l}}^{* *}\right)\right| \\
= & \frac{1}{d_{i_{l}}-\left(a_{i_{l} i_{l}}+\alpha_{i_{l}}^{-1} \beta_{i_{l}} B_{i_{l}}\right) l_{i_{l}, 2}} \\
& \times \mid \sum_{j \in \mathbb{N}_{2,2}} a_{i_{l} j} l_{j, 1}\left(x_{j}^{*}-x_{j}^{* *}\right) \\
& +\sum_{j \in \mathbb{N}_{2,3} \cup \mathbb{N}_{3}}^{j \neq i_{l}} a_{i_{1} j} l_{j, 2}\left(x_{j}^{*}-x_{j}^{* *}\right) \mid \\
\leq & \frac{\sum_{j \in \mathbb{N}_{2,2}}\left|a_{i_{l} j} l_{j, 1}\right|+\sum_{j \in \mathbb{N}_{2,3} \cup \mathbb{N}_{3}, j \neq i_{l}}\left|a_{i_{l} j} l_{j, 2}\right|}{d_{i_{l}}-\left(a_{i_{l} i_{l}}+\alpha_{i_{l}}^{-1} \beta_{i_{l}} B_{i_{l}}\right) l_{i_{l}, 2}} \\
& \times\left|x_{i_{l}}^{*}-x_{i_{l}}^{* *}\right|<\left|x_{i_{l}}^{*}-x_{i_{l}}^{* *}\right| .
\end{aligned}
$$

By the above two inequalities, we can deduce that $x_{i}^{*}=x_{i}^{* *}$ for all $i \in \mathbb{N}_{2,2} \cup \mathbb{N}_{2,3} \cup \mathbb{N}_{3}$. That is, the fixed point of map $\Gamma$ is unique.
Theorem 10. Suppose that $\mathbb{N}_{1} \cup \mathbb{N}_{2} \cup \mathbb{N}_{3} \cup \mathbb{N}_{4}=\{1,2, \ldots, N\}$.

(i) If the following conditions

$$
\begin{gathered}
d_{i}-a_{i i} l_{i, 2}-\sum_{j \in \mathbb{N}_{2,2}}\left|a_{i j}\right|\left|l_{j, 1}\right| \\
-\sum_{\substack{j \in \mathbb{N}_{2,3} \cup \mathbb{N}_{3} \\
j \neq i}}\left|a_{i j}\right|\left|l_{j, 2}\right|-\left|B_{i}\right|>0, \\
\alpha_{i}-\beta_{i}\left|l_{i, 2}\right|>0
\end{gathered}
$$

hold for all $i \in \mathbb{N}_{2,3} \cup \mathbb{N}_{3}$, then system (2) with activation functions (3) has $2^{\sharp \mathbb{N}_{2}}$ locally stable equilibria.

(ii) Furthermore, if the following conditions

$$
\begin{gathered}
-d_{i}+a_{i i} l_{i, 1}-\sum_{\substack{j \in \mathbb{N}_{2,2} \\
j \neq i}}\left|a_{i j}\right|\left|l_{j, 1}\right| \\
-\sum_{j \in \mathbb{N}_{2,3} \cup \mathbb{N}_{3}}\left|a_{i j}\right|\left|l_{j, 2}\right|-\left|B_{i}\right|>0, \\
\alpha_{i}-\beta_{i}\left|l_{i, 1}\right|>0
\end{gathered}
$$

hold for all $i \in \mathbb{N}_{2,2}$, then the other $3^{\sharp \mathbb{N}_{2}}-2^{\sharp \mathbb{N}_{2}}$ equilibria are unstable.

Proof. In the following, we will discuss the dynamical behaviors of $3^{\sharp N_{2}}$ equilibria in two cases, respectively.

Case (i). Consider $\mathbb{N}_{2,2}=\emptyset$. In this case, $\Omega_{\delta}$ and $\widetilde{\Omega}_{\delta}$ can be rewritten as

$$
\begin{aligned}
& \Omega_{\delta}= \prod_{i \in \mathbb{N}_{1} \cup \mathbb{N}_{2,1}}\left(-\infty, p_{i}\right] \times \prod_{i \in \mathbb{N}_{2,3}}\left[r_{i},+\infty\right) \\
& \times \prod_{i \in \mathbb{N}_{3}}\left[r_{i}, q_{i}\right] \times \prod_{i \in \mathbb{N}_{4}}\left[q_{i},+\infty\right), \\
& \widetilde{\Omega}_{\delta}=\prod_{i \in \mathbb{N}_{1} \cup \mathbb{N}_{2,1}}\left(-\infty, p_{i}\right] \times \prod_{i \in \mathbb{N}_{2,3} \cup \mathbb{N}_{3}}\left[r_{i}, q_{i}\right] \times \prod_{i \in \mathbb{N}_{4}}\left[q_{i},+\infty\right) .
\end{aligned}
$$

From (53)-(54), we can choose a sufficiently small number $\eta>0$ such that

$$
\begin{gathered}
d_{i}-a_{i i} l_{i, 2}-\sum_{\substack{j \in \mathbb{N}_{2,3} \cup \mathbb{N}_{3} \\
j \neq i}}\left|a_{i j}\right|\left|l_{j, 2}\right|-\left|B_{i}\right|>\eta>0, \\
\alpha_{i}-\beta_{i}\left|l_{i, 2}\right|>\eta>0
\end{gathered}
$$

hold for all $i \in \mathbb{N}_{2,3} \cup \mathbb{N}_{3}$.

Let $\left(x^{T}(t), S^{T}(t)\right)^{T}$ be any a solution of system (2) with initial state $\left(x^{T}(0), S^{T}(0)\right)^{T} \in \widetilde{\Omega}_{\delta} \times \prod_{i=1}^{N}\left[\alpha_{i}^{-1} \beta_{i} u_{i}, \alpha_{i}^{-1} \beta_{i} v_{i}\right]$. From Lemmas 4-6 and the proof of Theorem 7, we get that there exists some $T_{0} \geq 0$ such that $\left(x^{T}\left(T_{0}\right), S^{T}\left(T_{0}\right)\right)^{T} \in$ $\widetilde{\Omega}_{\delta} \times \prod_{i=1}^{N}\left[\alpha_{i}^{-1} \beta_{i} u_{i}, \alpha_{i}^{-1} \beta_{i} v_{i}\right]$. By the positive invariance of 
$\widetilde{\Omega}_{\delta} \times \prod_{i=1}^{N}\left[\alpha_{i}^{-1} \beta_{i} u_{i}, \alpha_{i}^{-1} \beta_{i} v_{i}\right]$, we know that the solution $\left(x^{T}(t), S^{T}(t)\right)^{T} \in \widetilde{\Omega}_{\delta} \times \prod_{i=1}^{N}\left[\alpha_{i}^{-1} \beta_{i} u_{i}, \alpha_{i}^{-1} \beta_{i} v_{i}\right]$ for all $t \geq T_{0}$, and its dynamics can be described by

$$
\begin{aligned}
& \dot{x}_{i}(t)=-d_{i} x_{i}(t)+a_{i i} u_{i}+\sum_{\substack{j \in \mathbb{N}_{1} \cup \mathbb{N}_{2,1} \\
j \neq i}} a_{i j} u_{j} \\
& +\sum_{j \in \mathbb{N}_{2,3} \cup \mathbb{N}_{3}} a_{i j}\left(l_{j, 2} x_{j}(t)+c_{j, 2}\right) \\
& +\sum_{j \in \mathbb{N}_{4}} a_{i j} u_{j}+B_{i} S_{i}(t)+I_{i}, \quad i \in \mathbb{N}_{1} \cup \mathbb{N}_{2,1}, \\
& \dot{x}_{i}(t)=-d_{i} x_{i}(t)+a_{i i}\left(l_{i, 2} x_{i}(t)+c_{i, 2}\right) \\
& +\sum_{j \in \mathbb{N}_{1} \cup \mathbb{N}_{2,1}} a_{i j} u_{j}+\sum_{\substack{j \in \mathbb{N}_{2,3} \cup \mathbb{N}_{3} \\
j \neq i}} a_{i j}\left(l_{j, 2} x_{j}(t)+c_{j, 2}\right) \\
& +\sum_{j \in \mathbb{N}_{4}} a_{i j} u_{j}+B_{i} S_{i}(t)+I_{i}, \quad i \in \mathbb{N}_{2,3} \cup \mathbb{N}_{3}, \\
& \dot{x}_{i}(t)=-d_{i} x_{i}(t)+a_{i i} u_{i}+\sum_{j \in \mathbb{N}_{1} \cup \mathbb{N}_{2,1}} a_{i j} u_{j} \\
& +\sum_{j \in \mathbb{N}_{2,3} \cup \mathbb{N}_{3}} a_{i j}\left(l_{j, 2} x_{j}(t)+c_{j, 2}\right) \\
& +\sum_{\substack{j \in \mathbb{N}_{4} \\
j \neq i}} a_{i j} u_{j}+B_{i} S_{i}(t)+I_{i}, \quad i \in \mathbb{N}_{4}, \\
& \dot{S}_{i}(t)=-\alpha_{i} S_{i}(t)+\beta_{i} f_{i}\left(x_{i}(t)\right), \quad i=1,2, \ldots, N .
\end{aligned}
$$

We get from model (60) that

$$
\begin{aligned}
\frac{\mathrm{d}\left(x_{i}(t)-x_{i}^{*}\right)}{\mathrm{d} t}= & -d_{i}\left(x_{i}(t)-x_{i}^{*}\right) \\
& +\sum_{j \in \mathbb{N}_{2,3} \cup \mathbb{N}_{3}} a_{i j} l_{j, 2}\left(x_{j}(t)-x_{j}^{*}\right) \\
& +B_{i}\left(S_{i}(t)-S_{i}^{*}\right), \quad i \in \mathbb{N}_{1} \cup \mathbb{N}_{2,1} \cup \mathbb{N}_{4}, \\
\frac{\mathrm{d}\left(x_{i}(t)-x_{i}^{*}\right)}{\mathrm{d} t}= & \left(-d_{i}+a_{i i} l_{i, 2}\right)\left(x_{i}(t)-x_{i}^{*}\right) \\
& +\sum_{j \in \mathbb{N}_{2,3} \cup \mathbb{N}_{3}} a_{i j} l_{j, 2}\left(x_{j}(t)-x_{j}^{*}\right) \\
& +B_{i}\left(S_{i}(t)-S_{i}^{*}\right), \quad i \in \mathbb{N}_{2,3} \cup \mathbb{N}_{3}, \\
\frac{\mathrm{d}\left(S_{i}(t)-S_{i}^{*}\right)}{\mathrm{d} t}= & -\alpha_{i}\left(S_{i}(t)-S_{i}^{*}\right), \quad i \in \mathbb{N}_{1} \cup \mathbb{N}_{2,1} \cup \mathbb{N}_{4}, \\
\frac{\mathrm{d}\left(S_{i}(t)-S_{i}^{*}\right)}{\mathrm{d} t}= & -\alpha_{i}\left(S_{i}(t)-S_{i}^{*}\right) \\
& +\beta_{i} l_{i, 2}\left(x_{i}(t)-x_{i}^{*}\right), \quad i \in \mathbb{N}_{2,3} \cup \mathbb{N}_{3} .
\end{aligned}
$$

Let

$$
\begin{array}{r}
\varphi_{i}(t)=e^{\eta t}\left|x_{i}(t)-x_{i}^{*}\right|, \\
\psi_{i}(t)=e^{\eta t}\left|S_{i}(t)-S_{i}^{*}\right|, \\
i \in \mathbb{N}_{2,3} \cup \mathbb{N}_{3} ;
\end{array}
$$

then by using (61)-(62), we can derive that

$$
\begin{aligned}
D^{+} \varphi_{i}(t) & \\
= & \eta e^{\eta t}\left|x_{i}(t)-x_{i}^{*}\right|+e^{\eta t} \operatorname{sgn}\left(x_{i}(t)-x_{i}^{*}\right) \\
& \times\left[\left(-d_{i}+a_{i i} l_{i, 2}\right)\left(x_{i}(t)-x_{i}^{*}\right)\right. \\
& \left.+\sum_{\substack{j \in \mathbb{N}_{2,3} \cup \mathbb{N}_{3} \\
j \neq i}} a_{i j} l_{j, 2}\left(x_{j}(t)-x_{j}^{*}\right)+B_{i}\left(S_{i}(t)-S_{i}^{*}\right)\right] \\
\leq & \left(\eta-d_{i}+a_{i i} l_{i, 2}\right) \varphi_{i}(t) \\
& +\sum_{\substack{j \in \mathbb{N}_{2,3} \cup \mathbb{N}_{3} \\
j \neq i}}\left|a_{i j}\right|\left|l_{j, 2}\right| \varphi_{j}(t)+\left|B_{i}\right| \psi_{i}(t) .
\end{aligned}
$$

Similarly, we have

$$
\begin{aligned}
D^{+} \psi_{i}(t)= & \eta e^{\eta t}\left|S_{i}(t)-S_{i}^{*}\right|+e^{\eta t} \operatorname{sgn}\left(S_{i}(t)-S_{i}^{*}\right) \\
& \times\left[-\alpha_{i}\left(S_{i}(t)-S_{i}^{*}\right)+\beta_{i} l_{i, 2}\left(x_{i}(t)-x_{i}^{*}\right)\right] \\
\leq & \left(\eta-\alpha_{i}\right) \psi_{i}(t)+\beta_{i}\left|l_{i, 2}\right| \varphi_{i}(t) .
\end{aligned}
$$

Let $\sigma>0$ be an arbitrary real number and

$$
l_{0}=(1+\sigma) e^{\eta T_{0}}\left(\max _{1 \leq i \leq N}\left|x_{i}\left(T_{0}\right)-x_{i}^{*}\right|+\max _{1 \leq i \leq N}\left|S_{i}\left(T_{0}\right)-S_{i}^{*}\right|\right)
$$

then

$$
\begin{aligned}
\varphi_{i}\left(T_{0}\right) & =e^{\eta T_{0}}\left|x_{i}\left(T_{0}\right)-x_{i}^{*}\right| \\
& \leq e^{\eta T_{0}} \max _{1 \leq i \leq N}\left|x_{i}\left(T_{0}\right)-x_{i}^{*}\right|<l_{0}, \quad i \in \mathbb{N}_{2,3} \cup \mathbb{N}_{3}, \\
\psi_{i}\left(T_{0}\right) & =e^{\eta T_{0}}\left|S_{i}\left(T_{0}\right)-S_{i}^{*}\right| \\
& \leq e^{\eta T_{0}} \max _{1 \leq i \leq N}\left|S_{i}\left(T_{0}\right)-S_{i}^{*}\right|<l_{0}, \quad i \in \mathbb{N}_{2,3} \cup \mathbb{N}_{3} .
\end{aligned}
$$

In the following, we will prove that

$$
\varphi_{i}(t) \leq l_{0}, \quad \psi_{i}(t) \leq l_{0}, \quad t>T_{0}, i \in \mathbb{N}_{2,3} \cup \mathbb{N}_{3} .
$$


If (67) is not true, then there exists some $i \in \mathbb{N}_{2,3} \cup \mathbb{N}_{3}$ and $t^{*}>T_{0}$ such that either

$$
\begin{aligned}
& \varphi_{i}\left(t^{*}\right)=l_{0}, \quad D^{+} \varphi_{i}\left(t^{*}\right)>0, \\
& \varphi_{j}\left(t^{*}\right) \leq l_{0} \quad\left(T_{0} \leq t \leq t^{*}, j \in \mathbb{N}_{2,3} \cup \mathbb{N}_{3}\right), \\
& \psi_{j}\left(t^{*}\right) \leq l_{0} \quad\left(T_{0} \leq t \leq t^{*}, j \in \mathbb{N}_{2,3} \cup \mathbb{N}_{3}\right)
\end{aligned}
$$

or

$$
\begin{aligned}
& \psi_{i}\left(t^{*}\right)=l_{0}, \quad D^{+} \psi_{i}\left(t^{*}\right)>0, \\
& \psi_{j}\left(t^{*}\right) \leq l_{0} \quad\left(T_{0} \leq t \leq t^{*}, j \in \mathbb{N}_{2,3} \cup \mathbb{N}_{3}\right), \\
& \varphi_{j}\left(t^{*}\right) \leq l_{0} \quad\left(T_{0} \leq t \leq t^{*}, j \in \mathbb{N}_{2,3} \cup \mathbb{N}_{3}\right) .
\end{aligned}
$$

For the first case, it follows from (58) and (63) that

$$
\begin{aligned}
D^{+} \varphi_{i}\left(t^{*}\right) \leq & \left(\eta-d_{i}+a_{i i} l_{i, 2}\right) \varphi_{i}\left(t^{*}\right) \\
& +\sum_{\substack{j \in \mathbb{N}_{2,3} \cup \mathbb{N}_{3} \\
j \neq i}}\left|a_{i j}\right|\left|l_{j, 2}\right| \varphi_{j}\left(t^{*}\right)+\left|B_{i}\right| \psi_{i}\left(t^{*}\right) \\
\leq & {\left[\eta-d_{i}+a_{i i} l_{i, 2}+\sum_{\substack{j \in \mathbb{N}_{2,3} \cup \mathbb{N}_{3} \\
j \neq i}}\left|a_{i j}\right|\left|l_{j, 2}\right|+\left|B_{i}\right|\right] l_{0} } \\
< & 0,
\end{aligned}
$$

that is a contradiction. For the second case, by using (59) and (64), we get

$$
\begin{aligned}
D^{+} \psi_{i}\left(t^{*}\right) & \leq\left(\eta-\alpha_{i}\right) \psi_{i}\left(t^{*}\right)+\beta_{i}\left|l_{i, 2}\right| \varphi_{i}\left(t^{*}\right) \\
& \leq\left[\eta-\alpha_{i}+\beta_{i}\left|l_{i, 2}\right|\right] l_{0}<0,
\end{aligned}
$$

that is also a contradiction. So (67) holds; that is, $\left|x_{i}(t)-x_{i}^{*}\right| \leq$ $l_{0} e^{-\eta t},\left|S_{i}(t)-S_{i}^{*}\right| \leq l_{0} e^{-\eta t}$ hold for all $t \geq T_{0}$ and $i \in \mathbb{N}_{2,3} \cup \mathbb{N}_{3}$.

From (61), we derive that

$$
\begin{array}{r}
\left|S_{i}(t)-S_{i}^{*}\right|=\left|S_{i}\left(T_{0}\right)-S_{i}^{*}\right| e^{-\alpha_{i}\left(t-T_{0}\right)}, \\
t \geq T_{0}, \quad i \in \mathbb{N}_{1} \cup \mathbb{N}_{2,1} \cup \mathbb{N}_{4},
\end{array}
$$

which implies that $\lim _{t \rightarrow+\infty}\left|S_{i}(t)-S_{i}^{*}\right|=0\left(i \in \mathbb{N}_{1} \cup \mathbb{N}_{2,1} \cup \mathbb{N}_{4}\right)$. Thus, for any $\varepsilon>0$, there exists a constant $T_{1} \geq T_{0}$ such that

$$
\begin{array}{r}
\sum_{j \in \mathbb{N}_{2,3} \cup \mathbb{N}_{3}}\left|a_{i j}\right|\left|l_{j, 2}\right|\left|x_{j}(t)-x_{j}^{*}\right|+\left|B_{i}\right|\left|S_{i}(t)-S_{i}^{*}\right|<\varepsilon, \\
t \geq T_{1}, \quad i \in \mathbb{N}_{1} \cup \mathbb{N}_{2,1} \cup \mathbb{N}_{4} .
\end{array}
$$

Then we have

$$
\begin{array}{r}
D^{+}\left(\left|x_{i}(t)-x_{i}^{*}\right|\right) \leq-d_{i}\left|x_{i}(t)-x_{i}^{*}\right|+\varepsilon, \\
\quad i \in \mathbb{N}_{1} \cup \mathbb{N}_{2,1} \cup \mathbb{N}_{4},
\end{array}
$$

which implies that

$$
\begin{array}{r}
\left|x_{i}(t)-x_{i}^{*}\right| \leq e^{-d_{i}\left(t-T_{1}\right)}\left|x_{i}\left(T_{1}\right)-x_{i}^{*}\right|+ \\
\frac{\varepsilon}{d_{i}}\left(1-e^{-d_{i}\left(t-T_{1}\right)}\right), \\
\quad i \in \mathbb{N}_{1} \cup \mathbb{N}_{2,1} \cup \mathbb{N}_{4} .
\end{array}
$$

That is, $\lim _{t \rightarrow+\infty}\left|x_{i}(t)-x_{i}^{*}\right|=0\left(i \in \mathbb{N}_{1} \cup \mathbb{N}_{2,1} \cup \mathbb{N}_{4}\right)$.

To sum up, for all $i=1,2, \ldots, N$, we have $\lim _{t \rightarrow+\infty} \mid x_{i}(t)-$ $x_{i}^{*}\left|=0, \lim _{t \rightarrow+\infty}\right| S_{i}(t)-S_{i}^{*} \mid=0$. That is, the equilibrium point $\left(x^{* T}, S^{* T}\right)^{T}$ is locally stable in $\Omega_{\delta} \times$ $\prod_{i=1}^{N}\left[\alpha_{i}^{-1} \beta_{i} u_{i}, \alpha_{i}^{-1} \beta_{i} v_{i}\right]$.

Case (ii). Consider $\mathbb{N}_{2,2} \neq \emptyset$. In this case, let $\left(x^{T}(t), S^{T}(t)\right)^{T}$ be a solution of system (2) with initial condition $\left(x^{T}(0), S^{T}(0)\right)^{T}$ nearby $\left(x^{* T}, S^{* T}\right)^{T} \in \widetilde{\Omega}_{\delta} \times \prod_{i=1}^{N}\left[\alpha_{i}^{-1} \beta_{i} u_{i}, \alpha_{i}^{-1} \beta_{i} v_{i}\right]$. Without loss of generality, we assume that $\left(x^{T}(t), S^{T}(t)\right)^{T} \in \widetilde{\Omega}_{\delta} \times$ $\prod_{i=1}^{N}\left[\alpha_{i}^{-1} \beta_{i} u_{i}, \alpha_{i}^{-1} \beta_{i} v_{i}\right]$ for all $t \geq 0$. In fact, we can conclude that if the solution $\left(x^{T}(t), S^{T}(t)\right)^{T}$ gets out of region $\widetilde{\Omega}_{\delta} \times$ $\prod_{i=1}^{N}\left[\alpha_{i}^{-1} \beta_{i} u_{i}, \alpha_{i}^{-1} \beta_{i} v_{i}\right]$, the equilibrium point $\left(x^{* T}, S^{* T}\right)^{T}$ must be unstable according to the definition of instability. Thus, system (2) can be rewritten as

$$
\begin{aligned}
& \dot{x}_{i}(t)=-d_{i} x_{i}(t)+a_{i i} u_{i}+\sum_{\substack{j \in \mathbb{N}_{1} \cup \mathbb{N}_{2,1} \\
j \neq i}} a_{i j} u_{j} \\
& +\sum_{j \in \mathbb{N}_{2,2}} a_{i j}\left(l_{j, 1} x_{j}(t)+c_{j, 1}\right) \\
& +\sum_{j \in \mathbb{N}_{2,3} \cup \mathbb{N}_{3}} a_{i j}\left(l_{j, 2} x_{j}(t)+c_{j, 2}\right) \\
& +\sum_{j \in \mathbb{N}_{4}} a_{i j} u_{j}+B_{i} S_{i}(t)+I_{i}, \quad i \in \mathbb{N}_{1} \cup \mathbb{N}_{2,1}, \\
& \dot{x}_{i}(t)=-d_{i} x_{i}(t)+a_{i i}\left(l_{i, 1} x_{i}(t)+c_{i, 1}\right) \\
& +\sum_{j \in \mathbb{N}_{1} \cup \mathbb{N}_{2,1}} a_{i j} u_{j}+\sum_{\substack{j \in \mathbb{N}_{2,2} \\
j \neq i}} a_{i j}\left(l_{j, 1} x_{j}(t)+c_{j, 1}\right) \\
& +\sum_{j \in \mathbb{N}_{2,3} \cup \mathbb{N}_{3}} a_{i j}\left(l_{j, 2} x_{j}(t)+c_{j, 2}\right)+\sum_{j \in \mathbb{N}_{4}} a_{i j} u_{j} \\
& +B_{i} S_{i}(t)+I_{i}, \quad i \in \mathbb{N}_{2,2}, \\
& \dot{x}_{i}(t)=-d_{i} x_{i}(t)+a_{i i}\left(l_{i, 2} x_{i}(t)+c_{i, 2}\right) \\
& +\sum_{j \in \mathbb{N}_{1} \cup \mathbb{N}_{2,1}} a_{i j} u_{j}+\sum_{j \in \mathbb{N}_{2,2}} a_{i j}\left(l_{j, 1} x_{j}(t)+c_{j, 1}\right) \\
& +\sum_{\substack{j \in \mathbb{N}_{2,3} \cup \mathbb{N}_{3} \\
j \neq i}} a_{i j}\left(l_{j, 2} x_{j}(t)+c_{j, 2}\right)+\sum_{j \in \mathbb{N}_{4}} a_{i j} u_{j} \\
& +B_{i} S_{i}(t)+I_{i}, \quad i \in \mathbb{N}_{2,3} \cup \mathbb{N}_{3},
\end{aligned}
$$




$$
\begin{aligned}
\dot{x}_{i}(t)= & -d_{i} x_{i}(t)+a_{i i} u_{i}+\sum_{j \in \mathbb{N}_{1} \cup \mathbb{N}_{2,1}} a_{i j} u_{j} \\
& +\sum_{j \in \mathbb{N}_{2,2}} a_{i j}\left(l_{j, 1} x_{j}(t)+c_{j, 1}\right) \\
& +\sum_{j \in \mathbb{N}_{2,3} \cup \mathbb{N}_{3}} a_{i j}\left(l_{j, 2} x_{j}(t)+c_{j, 2}\right)+\sum_{\substack{j \in \mathbb{N}_{4} \\
j \neq i}} a_{i j} u_{j} \\
& +B_{i} S_{i}(t)+I_{i}, \quad i \in \mathbb{N}_{4}, \\
\dot{S}_{i}(t)= & -\alpha_{i} S_{i}(t)+\beta_{i} f_{i}\left(x_{i}(t)\right), \quad i=1,2, \ldots, N .
\end{aligned}
$$

It follows from model (76) that

$$
\begin{aligned}
& \frac{\mathrm{d}\left(x_{i}(t)-x_{i}^{*}\right)}{\mathrm{d} t}=-d_{i}\left(x_{i}(t)-x_{i}^{*}\right) \\
& +\sum_{j \in \mathbb{N}_{2,2}} a_{i j} l_{j, 1}\left(x_{j}(t)-x_{j}^{*}\right) \\
& +\sum_{j \in \mathbb{N}_{2,3} \cup \mathbb{N}_{3}} a_{i j} l_{j, 2}\left(x_{j}(t)-x_{j}^{*}\right) \\
& +B_{i}\left(S_{i}(t)-S_{i}^{*}\right), \quad i \in \mathbb{N}_{1} \cup \mathbb{N}_{2,1} \cup \mathbb{N}_{4}, \\
& \frac{\mathrm{d}\left(x_{i}(t)-x_{i}^{*}\right)}{\mathrm{d} t}=\left(-d_{i}+a_{i i} l_{i, 1}\right)\left(x_{i}(t)-x_{i}^{*}\right) \\
& +\sum_{\substack{j \in \mathbb{N}_{2,2} \\
j \neq i}} a_{i j} l_{j, 1}\left(x_{j}(t)-x_{j}^{*}\right) \\
& +\sum_{j \in \mathbb{N}_{2,3} \cup \mathbb{N}_{3}} a_{i j} l_{j, 2}\left(x_{j}(t)-x_{j}^{*}\right) \\
& +B_{i}\left(S_{i}(t)-S_{i}^{*}\right), \quad i \in \mathbb{N}_{2,2}, \\
& \frac{\mathrm{d}\left(x_{i}(t)-x_{i}^{*}\right)}{\mathrm{d} t}=\left(-d_{i}+a_{i i} l_{i, 2}\right)\left(x_{i}(t)-x_{i}^{*}\right) \\
& +\sum_{j \in \mathbb{N}_{2,2}} a_{i j} l_{j, 1}\left(x_{j}(t)-x_{j}^{*}\right) \\
& +\sum_{\substack{j \in \mathbb{N}_{2,3} \cup \mathbb{N}_{3} \\
j \neq i}} a_{i j} l_{j, 2}\left(x_{j}(t)-x_{j}^{*}\right) \\
& +B_{i}\left(S_{i}(t)-S_{i}^{*}\right), \quad i \in \mathbb{N}_{2,3} \cup \mathbb{N}_{3}, \\
& \frac{\mathrm{d}\left(S_{i}(t)-S_{i}^{*}\right)}{\mathrm{d} t}=-\alpha_{i}\left(S_{i}(t)-S_{i}^{*}\right), \quad i \in \mathbb{N}_{1} \cup \mathbb{N}_{2,1} \cup \mathbb{N}_{4} \text {, } \\
& \frac{\mathrm{d}\left(S_{i}(t)-S_{i}^{*}\right)}{\mathrm{d} t}=-\alpha_{i}\left(S_{i}(t)-S_{i}^{*}\right)+\beta_{i} l_{i, 1}\left(x_{i}(t)-x_{i}^{*}\right), \\
& i \in \mathbb{N}_{2,2} \text {, } \\
& \frac{\mathrm{d}\left(S_{i}(t)-S_{i}^{*}\right)}{\mathrm{d} t}=-\alpha_{i}\left(S_{i}(t)-S_{i}^{*}\right)+\beta_{i} l_{i, 2}\left(x_{i}(t)-x_{i}^{*}\right), \\
& i \in \mathbb{N}_{2,3} \cup \mathbb{N}_{3} \text {. }
\end{aligned}
$$

Pick an initial state $\left(x^{T}(0), S^{T}(0)\right)^{T}$ of system (2) such that $\max _{1 \leq i \leq N}\left\{\left|x_{i}(0)-x_{i}^{*}\right|,\left|S_{i}(0)-S_{i}^{*}\right|\right\}=\max _{i \in \mathbb{N}_{2,2}}\left|x_{i}(0)-x_{i}^{*}\right|>0$.

In the following, we claim that

$$
\max _{i \in \mathbb{N}_{2,2} \cup \mathbb{N}_{2,3} \cup \mathbb{N}_{3}}\left\{\left|x_{i}(t)-x_{i}^{*}\right|,\left|S_{i}(t)-S_{i}^{*}\right|\right\}=\max _{i \in \mathbb{N}_{2,2}}\left|x_{i}(t)-x_{i}^{*}\right|
$$

holds for all $t \geq 0$. In fact, if (79) is not true, then there exists some $t_{0} \geq 0$ such that

$$
\begin{aligned}
\max & \left\{\max _{i \in \mathbb{N}_{2,3} \cup \mathbb{N}_{3}}\left|x_{i}\left(t_{0}\right)-x_{i}^{*}\right|, \max _{i \in \mathbb{N}_{2,2} \cup \mathbb{N}_{2,3} \cup \mathbb{N}_{3}}\left|S_{i}\left(t_{0}\right)-S_{i}^{*}\right|\right\} \\
& =\max _{i \in \mathbb{N}_{2,2}}\left|x_{i}\left(t_{0}\right)-x_{i}^{*}\right|
\end{aligned}
$$

then there exists some index $i_{0}$ such that one of the following three cases holds:

(a) $i_{0} \in \mathbb{N}_{2,3} \cup \mathbb{N}_{3}$ and $\left|x_{i_{0}}\left(t_{0}\right)-x_{i_{0}}^{*}\right|=\max _{i \in \mathbb{N}_{2,2}}\left|x_{i}\left(t_{0}\right)-x_{i}^{*}\right|$,

(b) $i_{0} \in \mathbb{N}_{2,3} \cup \mathbb{N}_{3}$ and $\left|S_{i_{0}}\left(t_{0}\right)-S_{i_{0}}^{*}\right|=\max _{i \in \mathbb{N}_{2,2}}\left|x_{i}\left(t_{0}\right)-x_{i}^{*}\right|$,

(c) $i_{0} \in \mathbb{N}_{2,2}$ and $\left|S_{i_{0}}\left(t_{0}\right)-S_{i_{0}}^{*}\right|=\max _{i \in \mathbb{N}_{2,2}}\left|x_{i}\left(t_{0}\right)-x_{i}^{*}\right|$.

For the case (a), it follows from (53) and (77) that

$$
\begin{aligned}
D^{+}\left(\left|x_{i_{0}}\left(t_{0}\right)-x_{i_{0}}^{*}\right|\right) \leq & \left(-d_{i_{0}}+a_{i_{0} i_{0}} l_{i_{0}, 2}\right)\left|x_{i_{0}}\left(t_{0}\right)-x_{i_{0}}^{*}\right| \\
& +\sum_{j \in \mathbb{N}_{2,2}}\left|a_{i_{0} j}\right| l_{j, 1}\left|x_{j}\left(t_{0}\right)-x_{j}^{*}\right| \\
& +\sum_{\substack{j \in \mathbb{N}_{2,3} \cup \mathbb{N}_{3} \\
j \neq i_{0}}}\left|a_{i_{0} j}\right|\left|l_{j, 2}\right|\left|x_{j}\left(t_{0}\right)-x_{j}^{*}\right| \\
& +\left|B_{i_{0}}\right|\left|S_{i_{0}}\left(t_{0}\right)-S_{i_{0}}^{*}\right| \\
\leq & \left(\begin{array}{l}
-d_{i_{0}}+a_{i_{0} i_{0}} l_{i_{0}, 2}+\sum_{j \in \mathbb{N}_{2,2}}\left|a_{i_{0} j}\right| l_{j, 1} \\
+\sum_{\substack{j \in \mathbb{N}_{2,3} \cup \mathbb{N}_{3} \\
j \neq i_{0}}}\left|a_{i_{0} j}\right|\left|l_{j, 2}\right|+\left|B_{i_{0}}\right|
\end{array}\right) \\
& \cdot\left|x_{i_{0}}\left(t_{0}\right)-x_{i_{0}}^{*}\right|<0 .
\end{aligned}
$$

For the case (b), by using (54) and (77), we get that

$$
D^{+}\left(\left|S_{i_{0}}\left(t_{0}\right)-S_{i_{0}}^{*}\right|\right) \leq\left(-\alpha_{i_{0}}+\beta_{i_{0}}\left|l_{i_{0}, 2}\right|\right)\left|S_{i_{0}}\left(t_{0}\right)-S_{i_{0}}^{*}\right|<0 .
$$

Similarly, for the case (c), by means of (56) and (77), we have

$$
D^{+}\left(\left|S_{i_{0}}\left(t_{0}\right)-S_{i_{0}}^{*}\right|\right) \leq\left(-\alpha_{i_{0}}+\beta_{i_{0}}\left|l_{i_{0}, 1}\right|\right)\left|S_{i_{0}}\left(t_{0}\right)-S_{i_{0}}^{*}\right|<0 \text {. }
$$


Meanwhile, denote $i_{1} \in \mathbb{N}_{2,2}$ such that $\left|x_{i_{1}}\left(t_{0}\right)-x_{i_{1}}^{*}\right|=$ $\max _{i \in \mathbb{N}_{2,2}}\left|x_{i}\left(t_{0}\right)-x_{i}^{*}\right|$; then we obtain from (55) and (77) that

$$
\begin{aligned}
D^{+}\left(\left|x_{i_{1}}\left(t_{0}\right)-x_{i_{1}}^{*}\right|\right) \geq & \left(-d_{i_{1}}+a_{i_{1} i_{1}} l_{i_{1}, 1}\right)\left|x_{i_{1}}\left(t_{0}\right)-x_{i_{1}}^{*}\right| \\
& -\sum_{\substack{j \in \mathbb{N}_{2,2} \\
j \neq i_{1}}}\left|a_{i_{1} j}\right| l_{j, 1}\left|x_{j}\left(t_{0}\right)-x_{j}^{*}\right| \\
& -\sum_{j \in \mathbb{N}_{2,3} \cup \mathbb{N}_{3}}\left|a_{i_{1} j}\right|\left|l_{j, 2}\right|\left|x_{j}\left(t_{0}\right)-x_{j}^{*}\right| \\
& -\left|B_{i_{1}}\right|\left|S_{i_{1}}\left(t_{0}\right)-S_{i_{1}}^{*}\right| \\
\geq & \left(-d_{i_{1}}+a_{i_{1} i_{1}} l_{i_{1}, 1}-\sum_{\substack{j \in \mathbb{N}_{2,2} \\
j \neq i_{1}}}\left|a_{i_{1} j}\right| l_{j, 1}\right. \\
& \left.-\sum_{j \in \mathbb{N}_{2,3} \cup \mathbb{N}_{3}}\left|a_{i_{1} j}\right|\left|l_{j, 2}\right|-\left|B_{i_{1}}\right|\right) \\
& \cdot\left|x_{i_{1}}\left(t_{0}\right)-x_{i_{1}}^{*}\right|>0 .
\end{aligned}
$$

Thus, combining inequalities (79) and (84) together gives

$$
\begin{aligned}
\max _{i \in \mathbb{N}_{2,2} \cup \mathbb{N}_{2,3} \cup \mathbb{N}_{3}}\left\{\left|x_{i}(t)-x_{i}^{*}\right|,\left|S_{i}(t)-S_{i}^{*}\right|\right\} \\
=\max _{i \in \mathbb{N}_{2,2}}\left|x_{i}(t)-x_{i}^{*}\right| \geq \max _{i \in \mathbb{N}_{2,2}}\left|x_{i}(0)-x_{i}^{*}\right|>0
\end{aligned}
$$

which implies that

$$
\begin{gathered}
\max _{i \in \mathbb{N}_{2,2} \cup \mathbb{N}_{2,3} \cup \mathbb{N}_{3}}\left|x_{i}(t)-x_{i}^{*}\right|=\max _{i \in \mathbb{N}_{2,2}}\left|x_{i}(t)-x_{i}^{*}\right| \\
\geq \max _{i \in \mathbb{N}_{2,2}}\left|x_{i}(0)-x_{i}^{*}\right|
\end{gathered}
$$

holds for all $t \geq 0$.

Therefore, there must exist an index $i_{2} \in \mathbb{N}_{2,2}$ and an increasing time sequence $\left\{t_{k}\right\}_{k=1}^{\infty}$ with $\lim _{t \rightarrow+\infty} t_{k}=+\infty$ such that $\left|x_{i_{2}}\left(t_{k}\right)-x_{i_{2}}^{*}\right| \geq \max _{i \in \mathbb{N}_{22}}\left|x_{i}\left(t_{0}\right)-x_{i}^{*}\right|$. That is, $x_{i_{2}}(t)$ would not converge to $x_{i}^{*}$ when $t$ tends to $+\infty$. In other words, the equilibrium point $x^{*}$ is unstable.

In the following, we will consider the dynamical behaviors of system (2) with activation functions (4). Note that $\max \left\{-a_{i j}, a_{i j}\right\}=\left|a_{i j}\right|$ and $\min \left\{-a_{i j}, a_{i j}\right\}=-\left|a_{i j}\right|$; in this case, $\mathbb{N}_{1}-\mathbb{N}_{4}$ reduce to the following index subsets:

$$
\begin{aligned}
& \overline{\mathbb{N}}_{1}=\left\{i: I_{i}<-\left|-d_{i}+a_{i i}\right|-\sum_{j \neq i, j=1}^{N}\left|a_{i j}\right|-\alpha_{i}^{-1} \beta_{i}\left|B_{i}\right|\right\}, \\
& \overline{\mathbb{N}}_{2}=\left\{i:\left|I_{i}\right|<-d_{i}+a_{i i}-\sum_{j \neq i, j=1}^{N}\left|a_{i j}\right|-\alpha_{i}^{-1} \beta_{i}\left|B_{i}\right|\right\},
\end{aligned}
$$

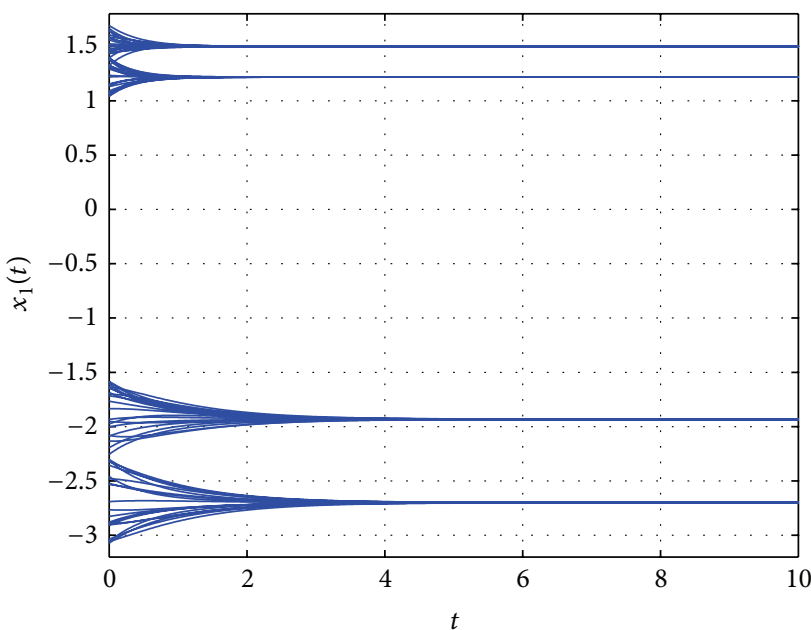

Figure 2: Transient behavior of $x_{1}$ in Example 1.

$$
\begin{aligned}
\overline{\mathbb{N}}_{3}=\left\{i:\left|-d_{i}+a_{i i}\right|+\sum_{j \neq i, j=1}^{N}\left|a_{i j}\right|+\alpha_{i}^{-1} \beta_{i}\left|B_{i}\right|\right. \\
\left.\quad<I_{i}<3 d_{i}+a_{i i}-\sum_{j \neq i, j=1}^{N}\left|a_{i j}\right|-\alpha_{i}^{-1} \beta_{i}\left|B_{i}\right|\right\},
\end{aligned}
$$

$$
\begin{aligned}
\overline{\mathbb{N}}_{4}=\{i: & I_{i}>\max \left\{3 d_{i}+a_{i i}, d_{i}-a_{i i}\right\} \\
& \left.+\sum_{j \neq i, j=1}^{N}\left|a_{i j}\right|+\alpha_{i}^{-1} \beta_{i}\left|B_{i}\right|\right\} .
\end{aligned}
$$

Meanwhile, conditions (47)-(48) become

$$
\begin{array}{r}
\sum_{\substack{j \in \mathbb{N}_{2,2} \\
j \neq i}}\left|a_{i j}\right|+\sum_{j \in \mathbb{N}_{2,3} \cup \mathbb{N}_{3}}\left|a_{i j}\right|<-d_{i}+a_{i i}+\alpha_{i}^{-1} B_{i} \beta_{i}, \\
i \in \mathbb{N}_{2,2}, \\
\sum_{j \in \mathbb{N}_{2,2}}\left|a_{i j}\right|+\sum_{\substack{j \in \mathbb{N}_{2,3} \cup \mathbb{N}_{3} \\
j \neq i}}\left|a_{i j}\right|<d_{i}+a_{i i}+\alpha_{i}^{-1} B_{i} \beta_{i}, \\
i \in \mathbb{N}_{2,3} \cup \mathbb{N}_{3} .
\end{array}
$$

Remark 11. Under index subsets $\overline{\mathbb{N}}_{2}-\overline{\mathbb{N}}_{3}$, conditions (88)-(89) always hold.

Proof. From the definition of index subset $\overline{\mathbb{N}}_{2}$, we get

$$
\sum_{j \neq i, j=1}^{N}\left|a_{i j}\right|<-d_{i}+a_{i i}+\alpha_{i}^{-1} \beta_{i}\left|B_{i}\right|<d_{i}+a_{i i}+\alpha_{i}^{-1} \beta_{i}\left|B_{i}\right| .
$$

Therefore, inequalities (88) and (89) hold for $i \in \mathbb{N}_{2,2}$ and $i \in \mathbb{N}_{2,3}$, respectively. 


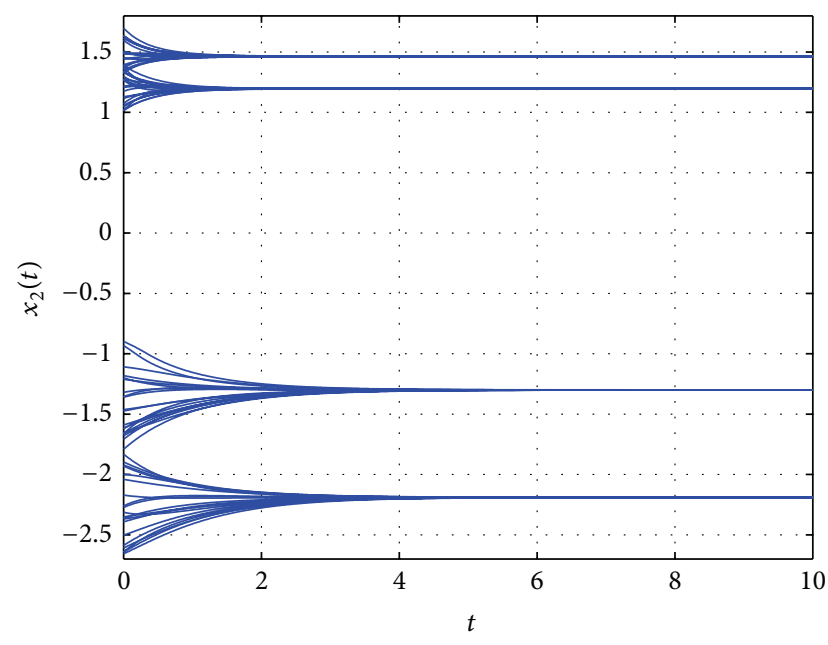

Figure 3: Transient behavior of $x_{2}$ in Example 1.
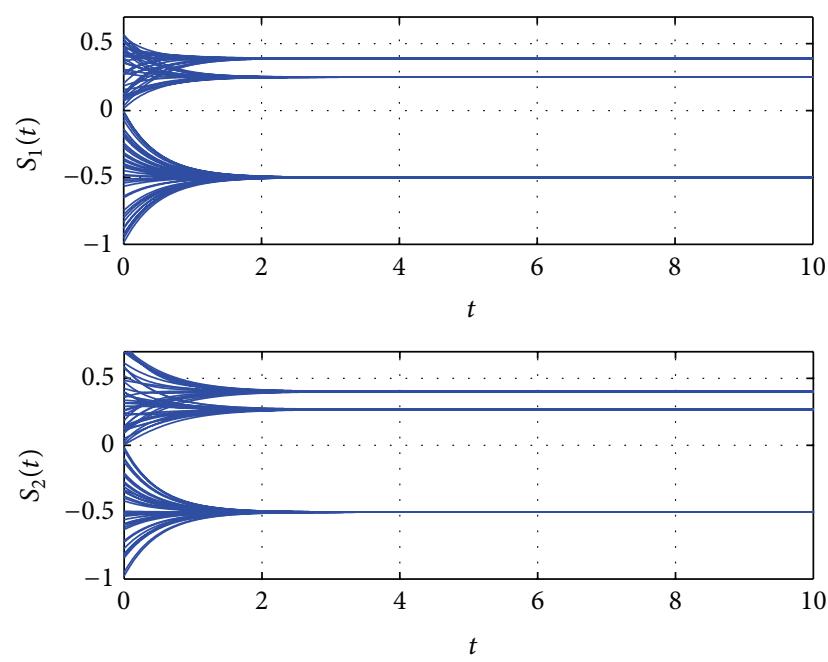

FIgURE 4: Transient behavior of $S_{1}$ and $S_{2}$ in Example 1.

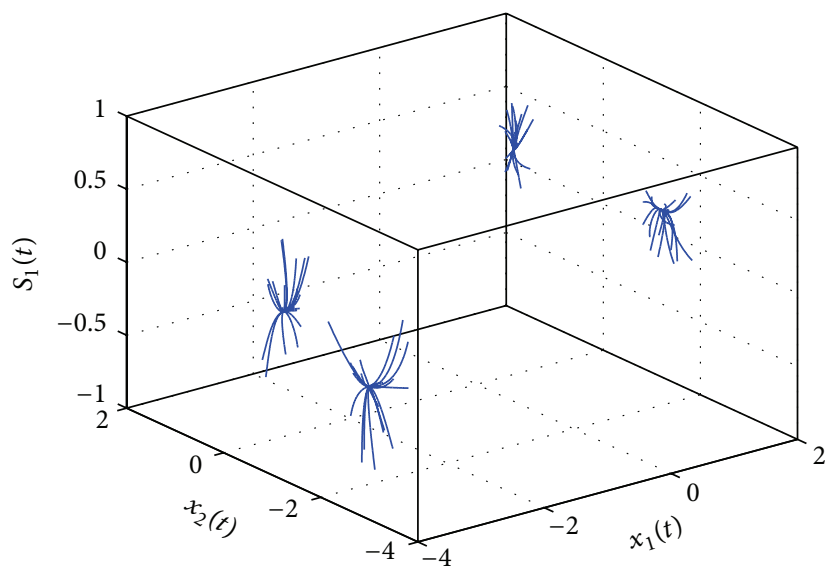

FIGURE 5: Phase plot of state variable $\left(x_{1}, x_{2}, S_{1}\right)^{T}$ in Example 1.

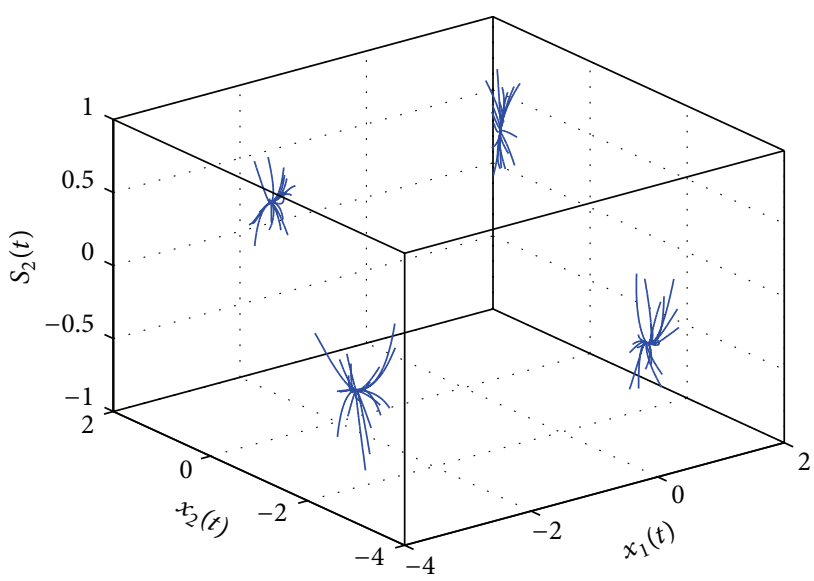

FIgURE 6: Phase plot of state variable $\left(x_{1}, x_{2}, S_{2}\right)^{T}$ in Example 1.
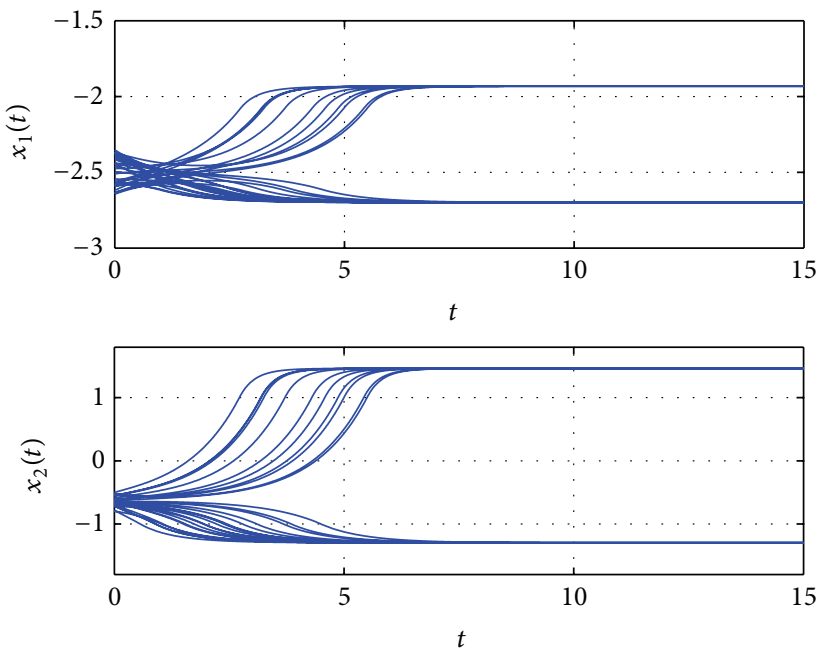

FIGURE 7: Transient behavior of $x_{1}$ and $x_{2}$ near the equilibrium point $(-201 / 80,-5 / 8,-1 / 2,-5 / 16)^{T}$ in Example 1.
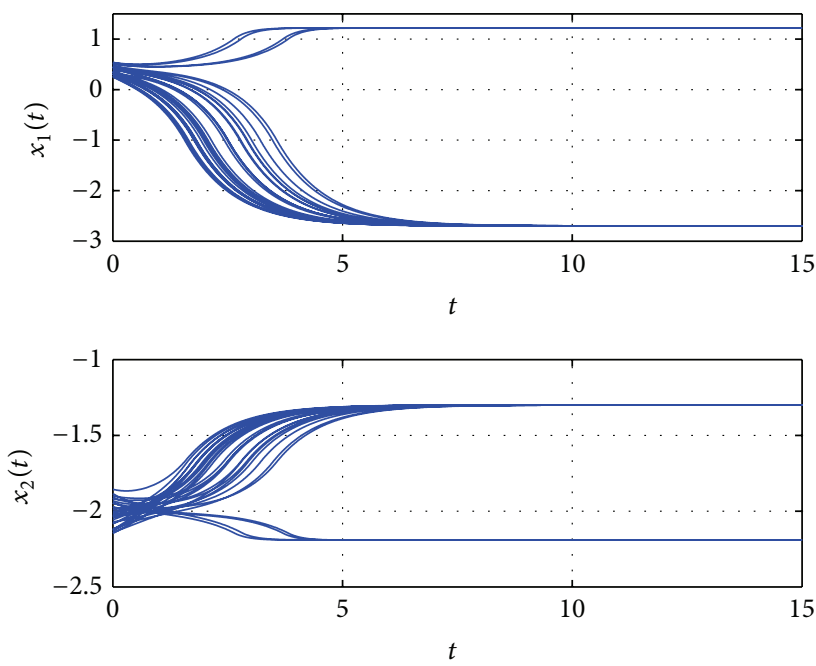

FIGURE 8: Transient behavior of $x_{1}$ and $x_{2}$ near the equilibrium point $(5 / 12,-241 / 120,5 / 24,-1 / 2)^{T}$ in Example 1. 

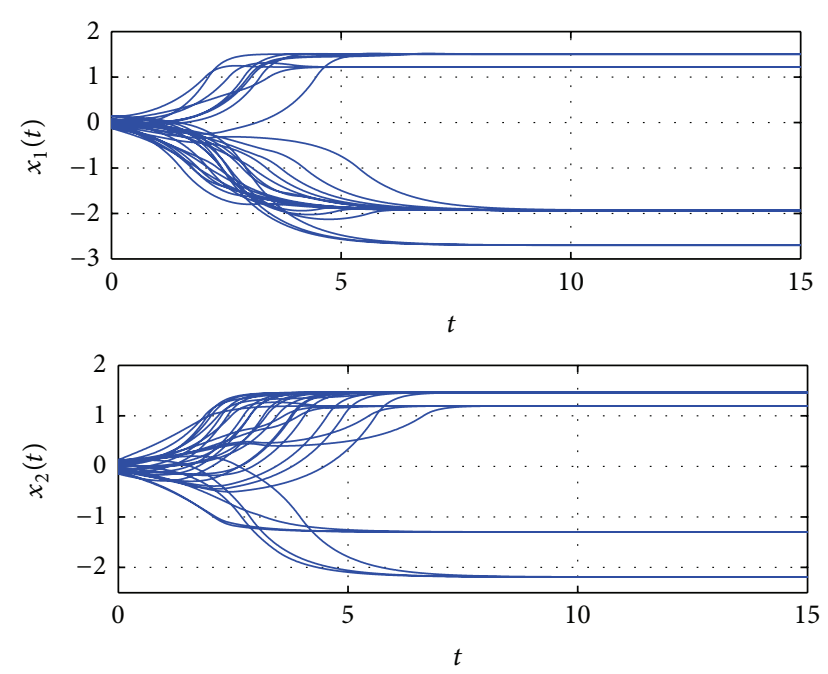

FIGURE 9: Transient behavior of $x_{1}$ and $x_{2}$ near the equilibrium point $(0,0,0,0)^{T}$ in Example 1 .
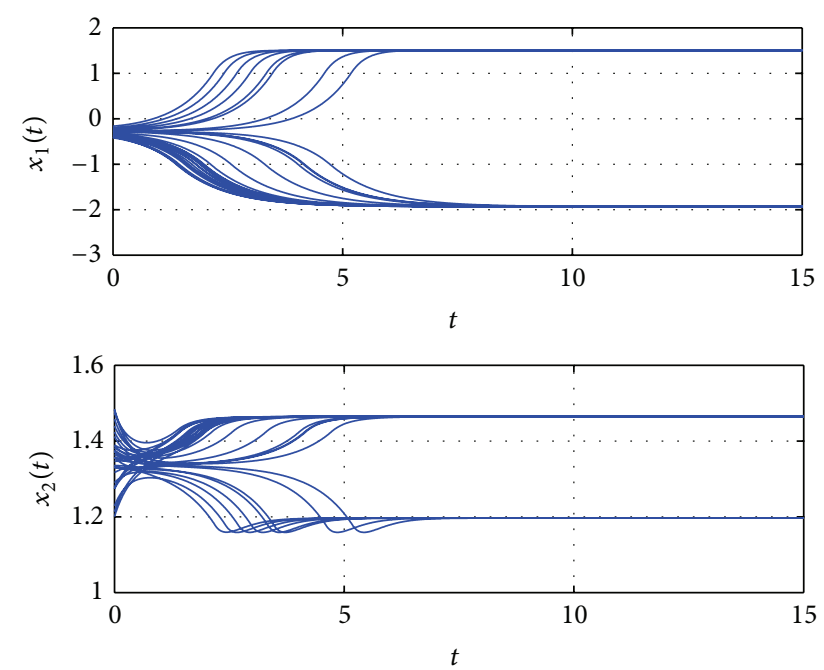

FIgURE 10: Transient behavior of $x_{1}$ and $x_{2}$ near the equilibrium point $(-100 / 361,482 / 361,-50 / 361,120 / 361)^{T}$ in Example 1.

Similarly, it follows from the definition of $\overline{\mathbb{N}}_{3}$ that

$$
\begin{aligned}
d_{i}- & a_{i i}+\sum_{j \neq i, j=1}^{N}\left|a_{i j}\right|-\alpha_{i}^{-1} \beta_{i} B_{i}<3 d_{i}+a_{i i} \\
& -\sum_{j \neq i, j=1}^{N}\left|a_{i j}\right|+\alpha_{i}^{-1} \beta_{i} B_{i},
\end{aligned}
$$

which implies that

$$
\sum_{j \neq i, j=1}^{N}\left|a_{i j}\right|<d_{i}+a_{i i}+\alpha_{i}^{-1} \beta_{i} B_{i} .
$$

Therefore, condition (89) holds for $i \in \mathbb{N}_{3}$.

From Theorem 10 and Remark 11, we can obtain Corollary 12 as follows.
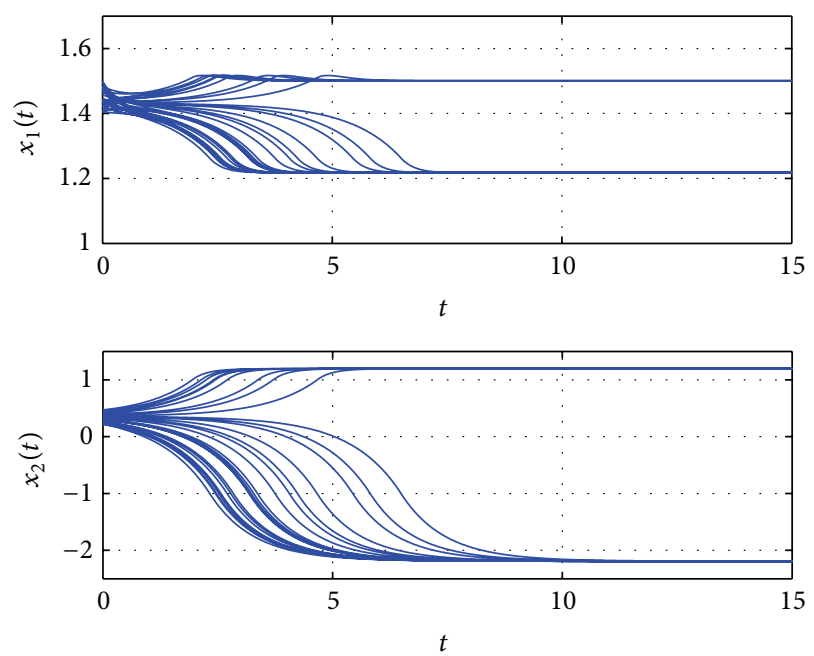

FIGURE 11: Transient behavior of $x_{1}$ and $x_{2}$ near the equilibrium point $(402 / 281,100 / 281,80 / 281,50 / 281)^{T}$ in Example 1.

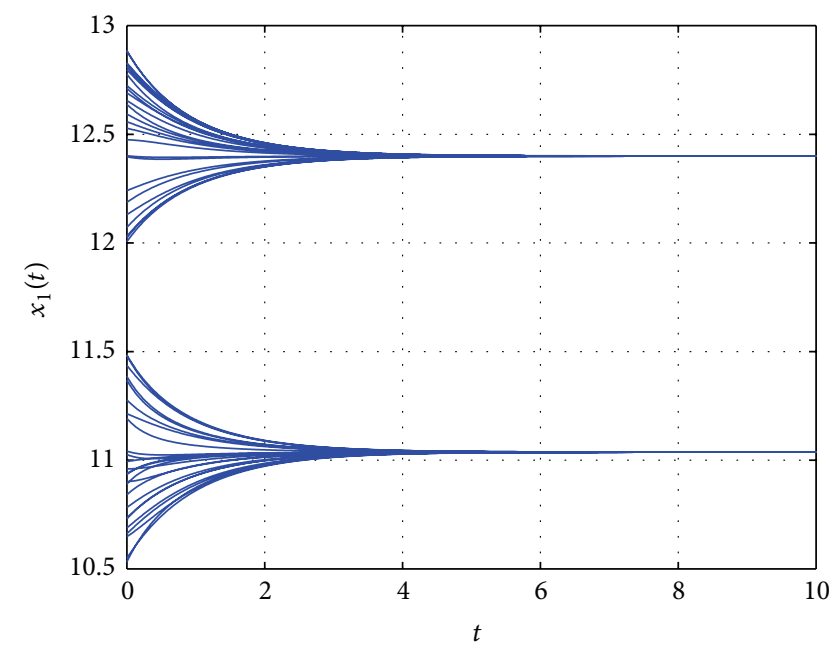

FIgURE 12: Transient behavior of $x_{1}$ in Example 2.

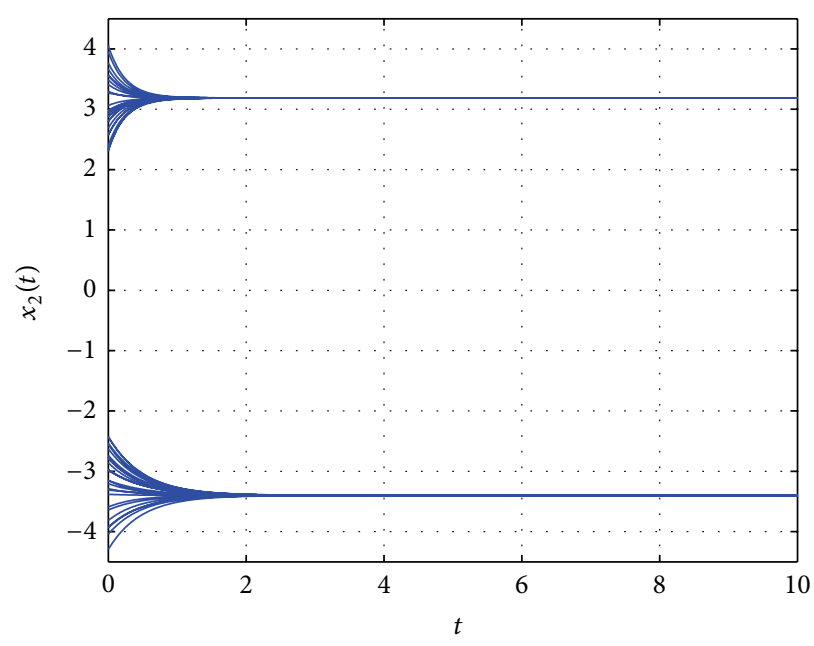

FIgURE 13: Transient behavior of $x_{2}$ in Example 2. 

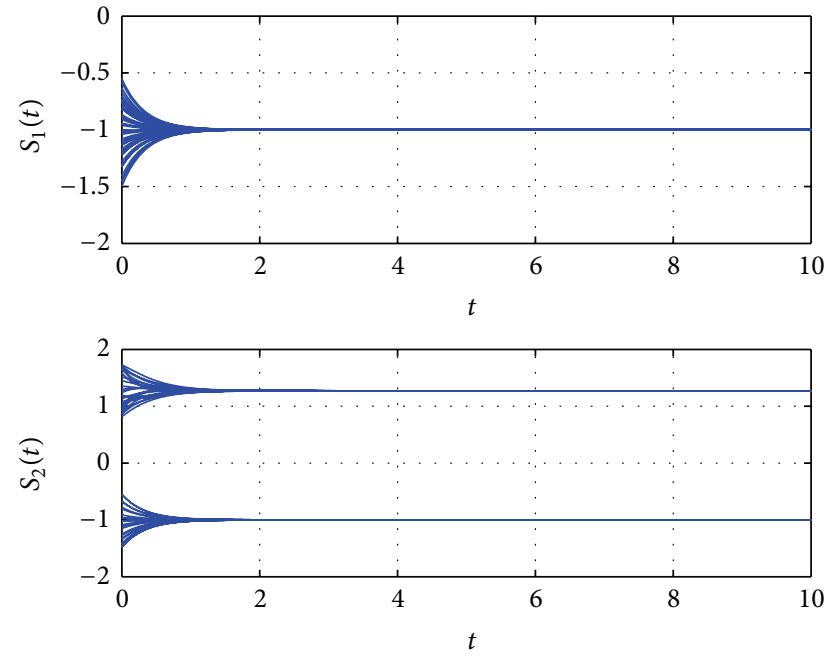

Figure 14: Transient behavior of $S_{1}$ and $S_{2}$ in Example 2.

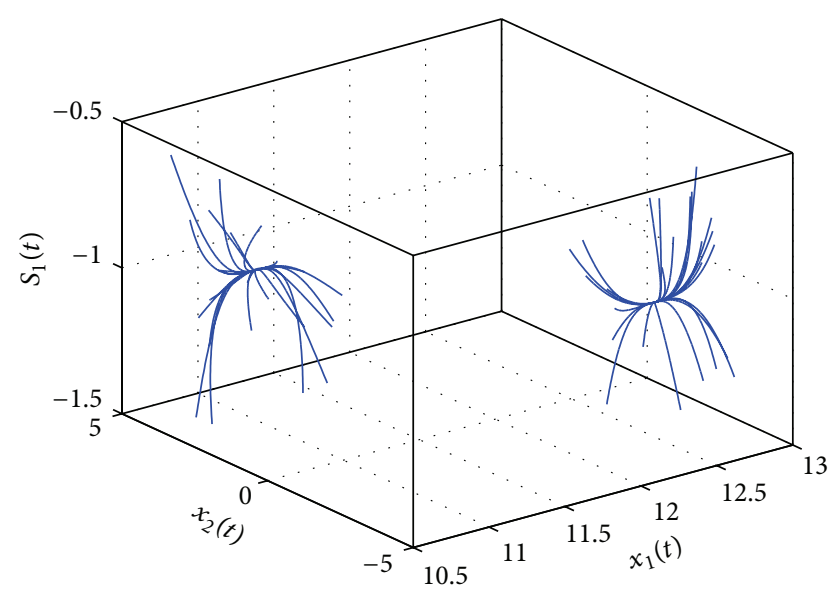

FIgURE 15: Phase plot of state variable $\left(x_{1}, x_{2}, S_{1}\right)^{T}$ in Example 2.

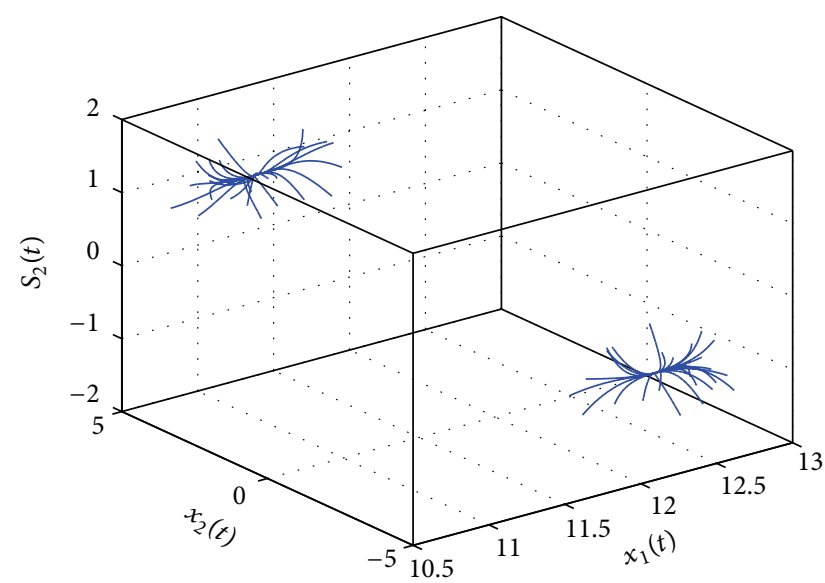

FIGURE 16: Phase plot of state variable $\left(x_{1}, x_{2}, S_{2}\right)^{T}$ in Example 2.
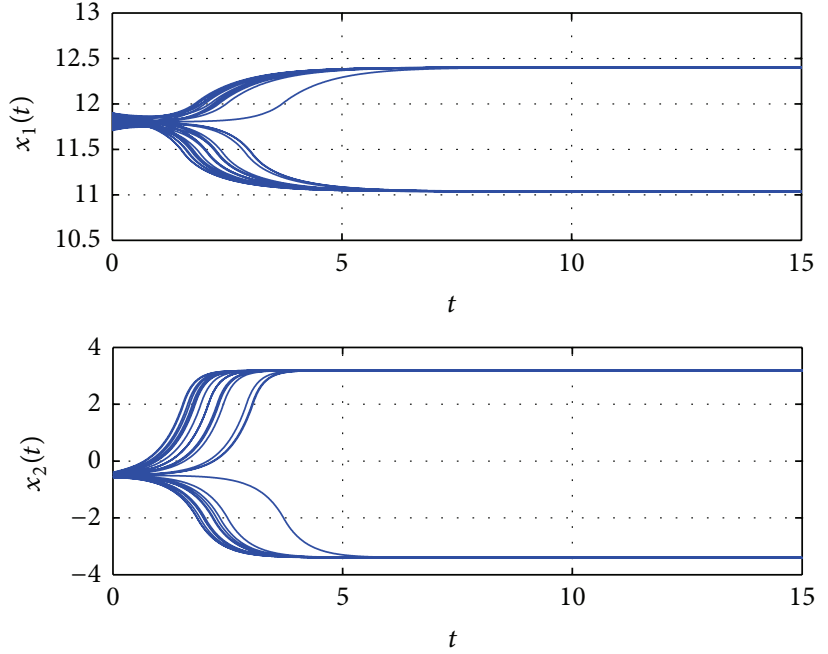

FIGURE 17: Transient behavior of $x_{1}$ and $x_{2}$ near the equilibrium point $(59 / 5,-1 / 2,-1,0)^{T}$ in Example 2 .

Corollary 12. Suppose that $\overline{\mathbb{N}}_{1} \cup \overline{\mathbb{N}}_{2} \cup \overline{\mathbb{N}}_{3} \cup \overline{\mathbb{N}}_{4}=\{1,2, \ldots, N\}$. If the following conditions

$$
\begin{gathered}
-d_{i}+a_{i i}-\sum_{\substack{j \in \overline{\mathbb{N}}_{2,2} \\
j \neq i}}\left|a_{i j}\right|-\sum_{j \in \overline{\mathbb{N}}_{2,3} \cup \overline{\mathbb{N}}_{3}}\left|a_{i j}\right|-\left|B_{i}\right|>0, \quad i \in \overline{\mathbb{N}}_{2,2} \\
d_{i}+a_{i i}-\sum_{j \in \overline{\mathbb{N}}_{2,2}}\left|a_{i j}\right|-\sum_{\substack{j \in \overline{\mathbb{N}}_{2,3} \cup \overline{\mathbb{N}}_{3} \\
j \neq i}}\left|a_{i j}\right|-\left|B_{i}\right|>0 \\
\alpha_{i}>\beta_{i}, \quad i \in \overline{\mathbb{N}}_{2,2} \cup \overline{\mathbb{N}}_{2,3} \cup \overline{\mathbb{N}}_{3}
\end{gathered}
$$

hold, then system (2) with activation functions (4) has exactly $3^{\sharp \mathbb{N}_{2}}$ equilibria, $2^{\sharp \mathbb{N}_{2}}$ of them are locally stable and others are unstable.

Proof. First of all, according to Theorem 7 and Remark 11, the exact existence of $3^{\sharp \overline{\mathbb{N}}_{2}}$ equilibria for system (2) with activation functions (4) can be guaranteed under condition $\overline{\mathbb{N}}_{1} \cup \overline{\mathbb{N}}_{2} \cup \overline{\mathbb{N}}_{3} \cup \overline{\mathbb{N}}_{4}=\{1,2, \ldots, N\}$. In addition, it is easy to see that conditions (93)-(95) imply the conditions (53)-(56) with $l_{i, 1}=1, l_{i, 2}=-1$. From Theorem 10, we derive the result of Corollary 12.

Let $B_{i}=\beta_{i}=S_{i}(t)=0(i=1,2, \ldots, N)$; then system (2) is transformed into the following neural networks which are investigated in [33]:

$$
\dot{x}_{i}(t)=-d_{i} x_{i}(t)+\sum_{j=1}^{N} a_{i j} f_{j}\left(x_{j}(t)\right)+I_{i} .
$$


In this case, $\overline{\mathbb{N}}_{1}-\overline{\mathbb{N}}_{4}$ turn into the following index subsets defined in [33]:

$$
\begin{aligned}
& \widetilde{\mathbb{N}}_{1}=\left\{i: I_{i}<-\left|-d_{i}+a_{i i}\right|-\sum_{j \neq i, j=1}^{N}\left|a_{i j}\right|\right\}, \\
& \widetilde{\mathbb{N}}_{2}=\left\{i:\left|I_{i}\right|<-d_{i}+a_{i i}-\sum_{j \neq i, j=1}^{N}\left|a_{i j}\right|\right\}, \\
& \widetilde{\mathbb{N}}_{3}=\left\{i:\left|-d_{i}+a_{i i}\right|+\sum_{j \neq i, j=1}^{N}\left|a_{i j}\right|<I_{i}\right. \\
& \widetilde{\mathbb{N}}_{4}=\left\{\begin{array}{c}
\left.<d_{i}+a_{i i}-\sum_{j \neq i, j=1}^{N}\left|a_{i j}\right|\right\}, \\
\left.i: I_{i}>\max \left\{3 d_{i}+a_{i i}, d_{i}-a_{i i}\right\}+\sum_{j \neq i, j=1}^{N}\left|a_{i j}\right|\right\} .
\end{array}\right.
\end{aligned}
$$

Applying Corollary 12, we can obtain easily Corollary 13 as follows.

Corollary 13. Suppose that $\widetilde{\mathbb{N}}_{1} \cup \widetilde{\mathbb{N}}_{2} \cup \widetilde{\mathbb{N}}_{3} \cup \widetilde{\mathbb{N}}_{4}=\{1,2, \ldots, N\}$ holds. Then system (96) with activation functions (4) has exactly $3^{\sharp \mathbb{N}_{2}}$ equilibria, $2^{\sharp \mathbb{N}_{2}}$ of them are locally stable and others are unstable.

Proof. First of all, it follows from the definition of index subset $\widetilde{\mathbb{N}}_{2}$ that (93)-(94) with $B_{i}=0$ hold for $i \in \widetilde{\mathbb{N}}_{2,2}$ and $i \in \widetilde{\mathbb{N}}_{2,3}$, respectively. From the definition of $\widetilde{\mathbb{N}}_{3}$, we have

$$
d_{i}-a_{i i}+\sum_{j \neq i, j=1}^{N}\left|a_{i j}\right|<3 d_{i}+a_{i i}-\sum_{j \neq i, j=1}^{N}\left|a_{i j}\right|,
$$

which implies that

$$
d_{i}+a_{i i}-\sum_{j \neq i, j=1}^{N}\left|a_{i j}\right|>0
$$

that is, inequality (94) with $B_{i}=0$ holds for $i \in \widetilde{\mathbb{N}}_{3}$. Inequality (95) is obvious, due to $\alpha_{i}>0$ and $\beta_{i}=0$. From Corollary 12, we derive the result of Corollary 13 .

Remark 14. In this paper, we study the multistability and instability of CNNs with activation functions (3). The models are different from and more general than those in [33], and the considered activation functions (3) are also more general than those employed in [33]. Moreover, the index subsets defined in this paper are less restrictive than those defined in [33].

Remark 15. Compared with the results reported in [33], it can be seen that Corollary 13 above is consistent with Theorem 1 in [33]. That is, if we specialize the system and activation functions in Theorem 10 to those considered in [33], we can obtain the main result in [33]. Therefore, Theorem 10 extends and improves the main result in [33].

\section{Two Illustrative Examples}

For convenience, we consider the following two-dimensional CNNs:

$$
\begin{aligned}
& \dot{x}_{i}(t)=-d_{i} x_{i}(t)+\sum_{j=1}^{2} a_{i j} f_{j}\left(x_{j}(t)\right)+B_{i} S_{i}(t)+I_{i}, \\
& \dot{S}_{i}(t)=-\alpha_{i} S_{i}(t)+\beta_{i} f_{i}\left(x_{i}(t)\right), \quad i=1,2 .
\end{aligned}
$$

Example 1. For system (100), take $d_{1}=d_{2}=1, a_{11}=a_{22}=2$, $a_{12}=0.5, a_{21}=-0.5, B_{1}=0.4, B_{2}=-0.4, I_{1}=I_{2}=0$, $\alpha_{1}=\alpha_{2}=2, \beta_{1}=\beta_{2}=1$, and

$$
f_{i}(x)=\left\{\begin{array}{ll}
-1, & -\infty<x<-1, \\
x, & -1 \leq x \leq 1, \\
-x+2, & 1<x \leq 3 \\
-1, & 3<x<+\infty
\end{array} \quad(i=1,2)\right.
$$

It is easy to see that $\mathbb{N}_{2}=\{1,2\}$. In addition, by simple computations, we have

$$
\begin{aligned}
& -d_{1}+a_{11}-\left|a_{12}\right|-\left|B_{1}\right|=0.1>0 \\
& -d_{2}+a_{22}-\left|a_{21}\right|-\left|B_{2}\right|=0.1>0 .
\end{aligned}
$$

Therefore, the conditions in Corollary 12 hold. According to Corollary 12, system (100) has exactly $3^{2}=9$ equilibria, $2^{2}=4$ equilibria are locally stable and others are unstable. In fact, by direct computations, we can obtain the nine equilibria $(-2.7,-1.3,-0.5,-0.5)^{T}$, $(-201 / 80,-5 / 8,-1 / 2,-5 / 16)^{T}, \quad(-541 / 280,41 / 28,-1 / 2,15 /$ $56)^{T}, \quad(5 / 12,-241 / 120,5 / 24,-1 / 2)^{T}, \quad(0,0,0,0)^{T}, \quad(-100 /$ $361,482 / 361,-50 / 361,120 / 361)^{T},(39 / 32,-701 / 320,25 / 64$, $-1 / 2)^{T}, \quad(402 / 281,100 / 281,80 / 281,50 / 281)^{T}$, and $(1385 /$ $921,1102 / 921,457 / 1842,185 / 921)^{T}$. From Figures 2, $3,4,5$, and 6 , it can be seen that the four equilibria $(-2.7,-1.3,-0.5,-0.5)^{T}, \quad(-541 / 280,41 / 28,-1 / 2,15 / 56)^{T}$, $(39 / 32,-701 / 320,25 / 64,-1 / 2)^{T}$, and $(1385 / 921,1102 / 921$, $457 / 1842,185 / 921)^{T}$ are locally stable. Figures $7,8,9,10$, and 11 confirm that the others are unstable.

Example 2. For system (100), take $d_{1}=1, a_{11}=-1, a_{12}=$ $-0.2, B_{1}=0.2, d_{2}=a_{22}=2, a_{21}=1 / 3, B_{2}=-0.2, I_{1}=9$, $I_{2}=0, \alpha_{1}=\alpha_{2}=3, \beta_{1}=\beta_{2}=1$, and

$$
f_{i}(x)=\left\{\begin{array}{ll}
-3, & -\infty<x<-2, \\
2 x+1, & -2 \leq x \leq 2, \\
-x+7, & 2<x \leq 10, \\
-3, & 10<x<+\infty .
\end{array} \quad(i=1,2) .\right.
$$


It is easy to see that $\mathbb{N}_{2}=\{2\}, \mathbb{N}_{4}=\{1\}$. Herein, the parameters satisfy conditions in Remark 9 and Theorem 10:

$$
\begin{aligned}
& \left|a_{21}\right| \max \left\{\left|l_{1,1}\right|,\left|l_{1,2}\right|\right\} \\
& =\frac{2}{3}<\frac{28}{15}=\min \left\{\left(a_{22}+\alpha_{2}^{-1} B_{2} \beta_{2}\right) l_{2,1}-d_{2},\right. \\
& \left.d_{2}-\left(a_{22}+\alpha_{2}^{-1} B_{2} \beta_{2}\right) l_{2,2}\right\}, \\
& d_{2}-a_{22} l_{2,2}-\left|a_{21}\right| \max \left\{\left|l_{1,1}\right|,\left|l_{1,2}\right|\right\}=\frac{10}{3}>0, \\
& -d_{2}+a_{22} l_{2,1}-\left|a_{21}\right| \max \left\{\left|l_{1,1}\right|,\left|l_{1,2}\right|\right\}=\frac{4}{3}>0 .
\end{aligned}
$$

From Remark 9 and Theorem 10, it follows that the system has exactly three equilibria $(62 / 5,-17 / 5,-1,-1)^{T}$, $(59 / 5,-1 / 2,-1,0)^{T}$, and $(3256 / 295,188 / 59,-1,75 / 59)^{T}$; the first and the third equilibria are locally stable, while the second equilibrium point is unstable. From Figures 12, $13,14,15$, and 16 , it can be seen that the two equilibria $(62 / 5,-17 / 5,-1,-1)^{T}$ and $(3256 / 295,188 / 59,-1,75 / 59)^{T}$ are locally stable. Figure 17 confirms that the equilibrium point $(59 / 5,-1 / 2,-1,0)^{T}$ is unstable.

\section{Conclusions}

In this paper, the multistability and instability issues have been studied for CNNs with Mexican-hat-type activation functions. We showed that under some conditions, the system has $3^{\sharp N_{2}}$ equilibria, $2^{\sharp N_{2}}$ of them are locally stable and others are unstable. Two examples with their computer simulations were given to illustrate the effectiveness of the obtained results. Some thorough analyses are needed further. Here, we only treated neural networks without time delay, how about when time delays are presented? This needs to be investigated in the future.

\section{Conflict of Interests}

The authors declare that they have no conflict of interests regarding the publication of this paper.

\section{Acknowledgments}

This work was jointly supported by the National Natural Science Foundation of China under Grants no. 61203300, 61263020, and 11072059, the Specialized Research Fund for the Doctoral Program of Higher Education under Grants no. 20120092120029 and 20110092110017, the Natural Science Foundation of Jiangsu Province of China under Grants no. BK2012319 and BK2012741, the China Postdoctoral Science Foundation funded project under Grant no. 2012M511177, and the Innovation Foundation of Southeast University under Grant no. 3207012401.

\section{References}

[1] A. Meyer-Baese, S. S. Pilyugin, and Y. Chen, "Global exponential stability of competitive neural networks with different time scales," IEEE Transactions on Neural Networks, vol. 14, no. 3, pp. 716-719, 2003.

[2] H. Lu and Z. He, "Global exponential stability of delayed competitive neural networks with different time scales," Neural Networks, vol. 18, no. 3, pp. 243-250, 2005.

[3] H. Lu and S. Amari, "Global exponential stability of multitime scale competitive neural networks with nonsmooth functions," IEEE Transactions on Neural Networks, vol. 17, no. 5, pp. 1152$1164,2006$.

[4] X. Nie and J. Cao, "Exponential stability of competitive neural networks with time-varying and distributed delays," Proceedings of the Institution of Mechanical Engineers. Part I: Journal of Systems and Control Engineering, vol. 222, no. 6, pp. 583-594, 2008.

[5] J. Cao and Y. Wan, "Matrix measure strategies for stability and synchronization of inertial BAM neural network with time delays," Neural Networks, vol. 53, pp. 165-172, 2014.

[6] J. Cao, A. Alofi, A. Al-Mazrooei, and A. Elaiw, "Synchronization of switched interval networks and applications to chaotic neural networks," Abstract and Applied Analysis, vol. 2013, Article ID 940573, 11 pages, 2013.

[7] X. Yang, J. Cao, and Z. Yang, "Synchronization of coupled reaction-diffusion neural networks with time-varying delays via pinning-impulsive controller," SIAM Journal on Control and Optimization, vol. 51, pp. 3486-3510, 2013.

[8] Z. Zeng and J. Wang, "Multiperiodicity and exponential attractivity evoked by periodic external inputs in delayed cellular neural networks," Neural Computation, vol. 18, no. 4, pp. 848$870,2006$.

[9] Z. Zeng and J. Wang, "Multiperiodicity of discrete-time delayed neural networks evoked by periodic external inputs," IEEE Transactions on Neural Networks, vol. 17, no. 5, pp. 1141-1151, 2006.

[10] J. Cao, G. Feng, and Y. Wang, "Multistability and multiperiodicity of delayed Cohen-Grossberg neural networks with a general class of activation functions," Physica D, vol. 237, no. 13, pp. 1734-1749, 2008.

[11] K.-H. Lin and C.-W. Shih, "Multiple almost periodic solutions in nonautonomous delayed neural networks," Neural Computation, vol. 19, no. 12, pp. 3392-3420, 2007.

[12] L. Wang, W. Lu, and T. Chen, "Multistability and new attraction basins of almost-periodic solutions of delayed neural networks," IEEE Transactions on Neural Networks, vol. 20, no. 10, pp. 15811593, 2009.

[13] E. Kaslik and S. Sivasundaram, "Impulsive hybrid discretetime Hopfield neural networks with delays and multistability analysis," Neural Networks, vol. 24, no. 4, pp. 370-377, 2011.

[14] E. Kaslik and S. Sivasundaram, "Multiple periodic solutions in impulsive hybrid neural networks with delays," Applied Mathematics and Computation, vol. 217, no. 10, pp. 4890-4899, 2011.

[15] Z. Huang, Q. Song, and C. Feng, "Multistability in networks with self-excitation and high-order synaptic connectivity," IEEE Transactions on Circuits and Systems I: Regular Papers, vol. 57, no. 8, pp. 2144-2155, 2010. 
[16] X. Nie and J. Cao, "Multistability of second-order competitive neural networks with nondecreasing saturated activation functions," IEEE Transactions on Neural Networks, vol. 22, no. 11, pp. 1694-1708, 2011.

[17] X. Nie and Z. Huang, "Multistability and multiperiodicity of high-order competitive neural networks with a general class of activation functions," Neurocomputing, vol. 82, pp. 1-13, 2012.

[18] C.-Y. Cheng, K.-H. Lin, and C.-W. Shih, "Multistability in recurrent neural networks," SIAM Journal on Applied Mathematics, vol. 66, no. 4, pp. 1301-1320, 2006.

[19] C.-Y. Cheng, K.-H. Lin, and C.-W. Shih, "Multistability and convergence in delayed neural networks," Physica D, vol. 225, no. 1, pp. 61-74, 2007.

[20] X. Nie and J. Cao, "Multistability of competitive neural networks with time-varying and distributed delays," Nonlinear Analysis: Real World Applications, vol. 10, no. 2, pp. 928-942, 2009.

[21] C.-Y. Cheng and C.-W. Shih, "Complete stability in multistable delayed neural networks," Neural Computation, vol. 21, no. 3, pp. 719-740, 2009.

[22] G. Huang and J. Cao, "Delay-dependent multistability in recurrent neural networks," Neural Networks, vol. 23, no. 2, pp. 201209, 2010.

[23] L. Wang, W. Lu, and T. Chen, "Coexistence and local stability of multiple equilibria in neural networks with piecewise linear nondecreasing activation functions," Neural Networks, vol. 23, no. 2, pp. 189-200, 2010.

[24] Z. Zeng and W. Zheng, "Multistability of neural networks with time-varying delays and concaveconvex characteristics," IEEE Transactions on Neural Networks and Learning Systems, vol. 23, pp. 293-305, 2012.

[25] Z. Zeng, T. Huang, and W. X. Zheng, "Multistability of recurrent neural networks with time-varying delays and the piecewise linear activation function," IEEE Transactions on Neural Networks, vol. 21, no. 8, pp. 1371-1377, 2010.

[26] G. Huang and J. Cao, "Multistability in bidirectional associative memory neural networks," Physics Letters A, vol. 372, no. 16, pp. 2842-2854, 2008.

[27] Z. Huang, X. Wang, and C. Feng, "Multiperiodicity of periodically oscillated discrete-time neural networks with transient excitatory self-connections and sigmoidal nonlinearities," IEEE Transactions on Neural Networks, vol. 21, no. 10, pp. 1643-1655, 2010.

[28] W. Lu, L. Wang, and T. Chen, "On attracting basins of multiple equilibria of a class of cellular neural networks," IEEE Transactions on Neural Networks, vol. 22, no. 3, pp. 381-394, 2011.

[29] X. Nie, J. Cao, and S. Fei, "Multistability and instability of delayed competitive neural networks with nondecreasing piecewise linear activation functions," Neurocomputing, vol. 119, pp. 281-291, 2013.

[30] Y. Wang and J. Cao, "Multiperiodicity evoked by periodic external inputs in Cohen-Grossbergtype BAM networks with discrete and distributed delays," British Journal of Mathematics and Computer Science, vol. 2, pp. 94-113, 2012.

[31] J. Cao and Y. Wang, "Bi-periodicity evoked by periodic external inputs in delayed Cohen-Grossberg-type bidirectional associative memory networks," Physica Scripta, vol. 81, no. 5, Article ID 055803, 17 pages, 2010.

[32] G. Huang and J. Cao, "Multistability of neural networks with discontinuous activation function," Communications in Nonlinear Science and Numerical Simulation, vol. 13, no. 10, pp. 22792289, 2008.
[33] L. Wang and T. Chen, "Multistability of neural networks with Mexican-hat-type activation functions," IEEE Transactions on Neural Networks and Learning Systems, vol. 23, pp. 1816-1826, 2012. 


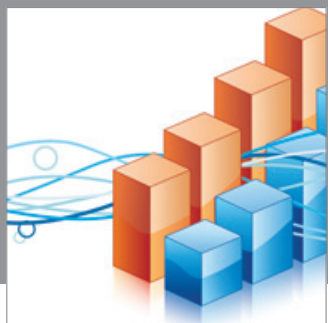

Advances in

Operations Research

mansans

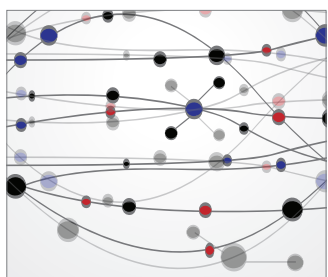

The Scientific World Journal
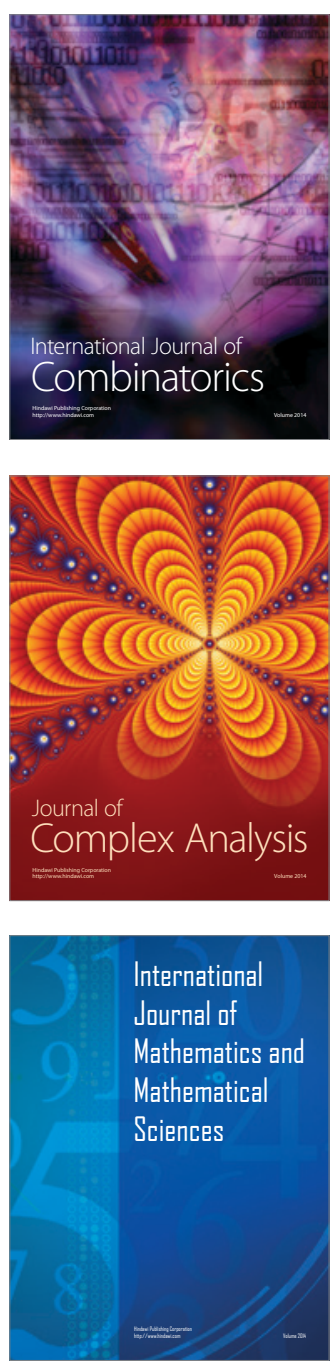
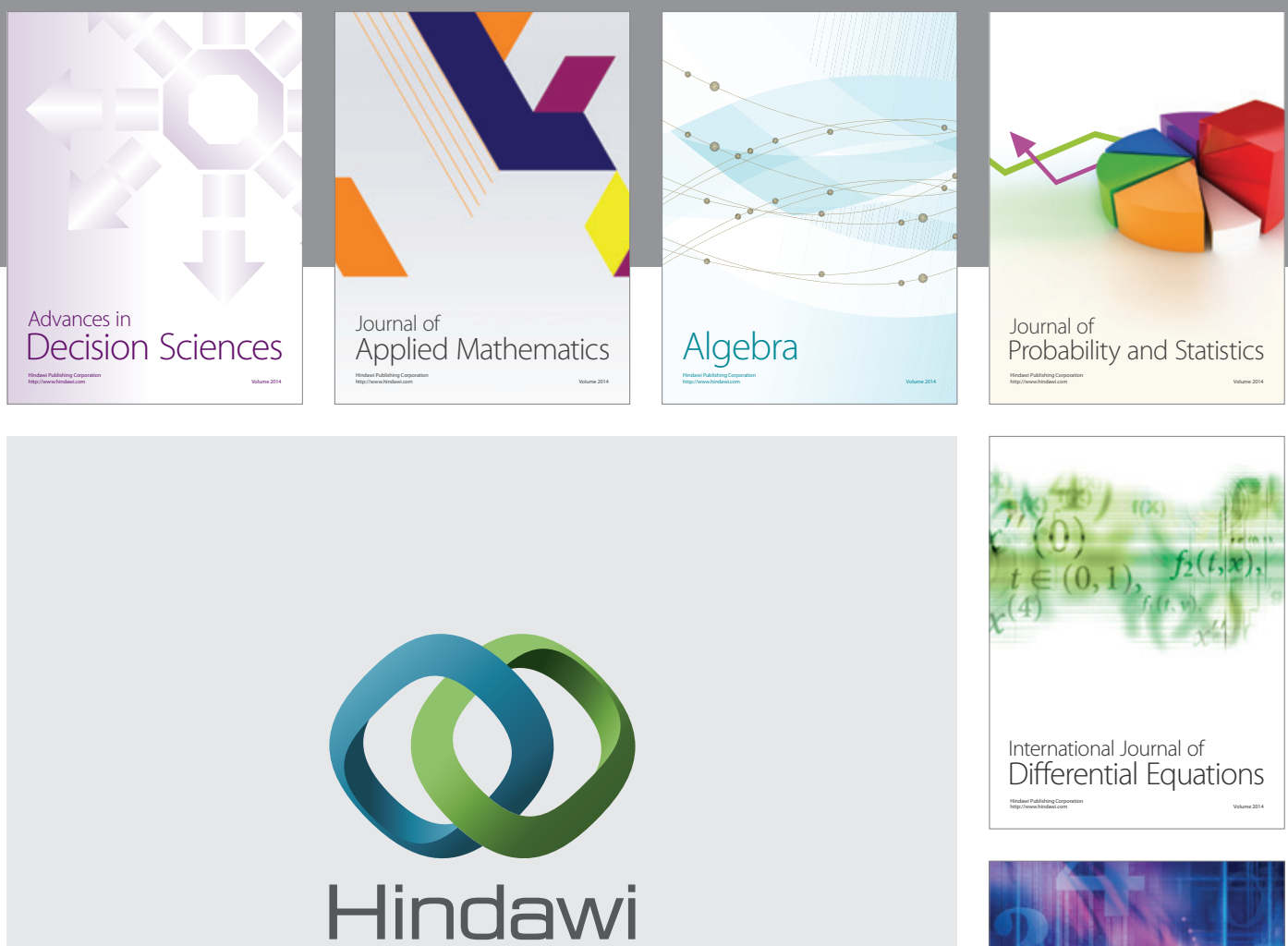

Submit your manuscripts at http://www.hindawi.com
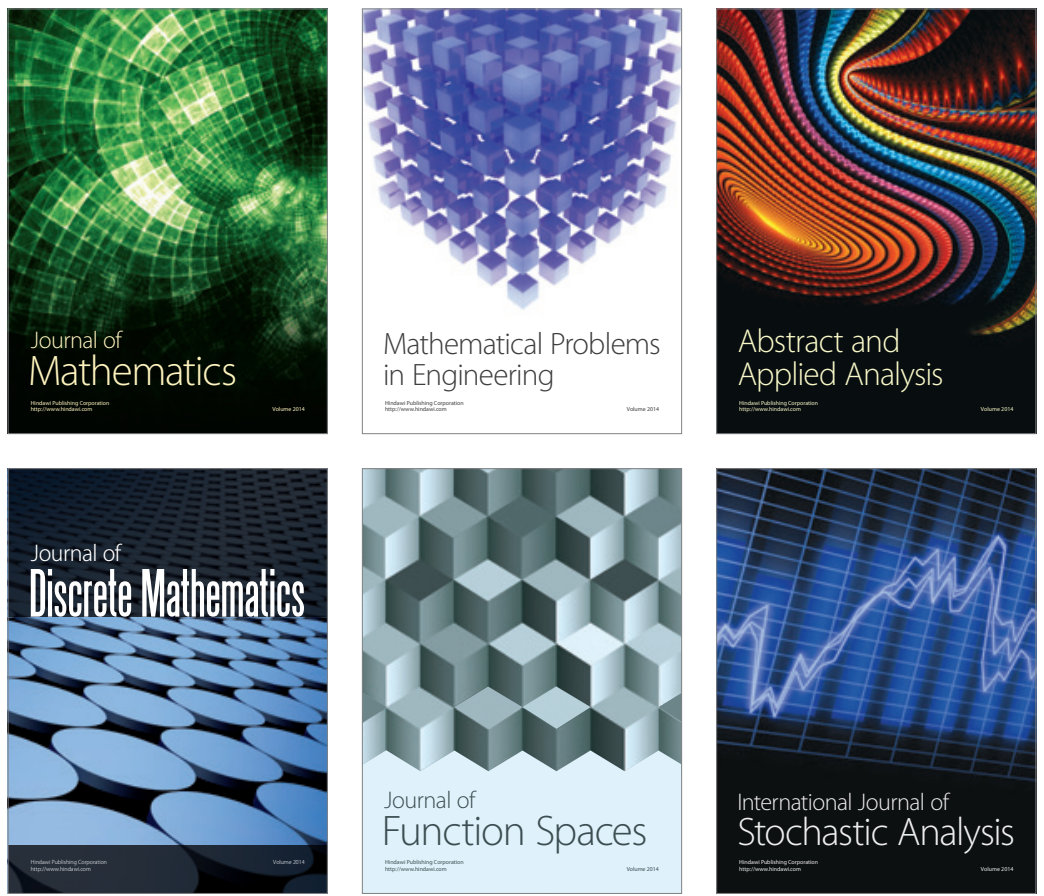

Journal of

Function Spaces

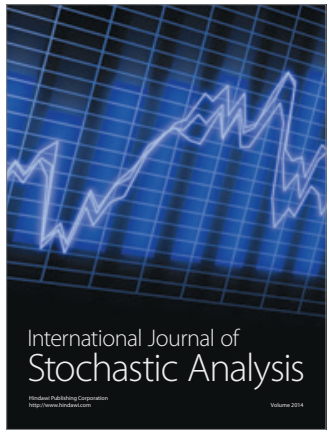

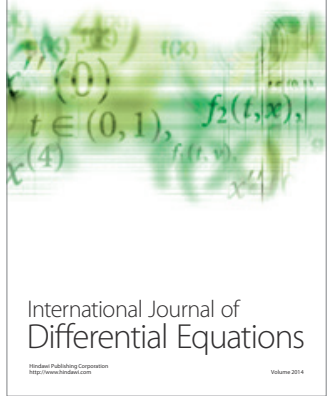
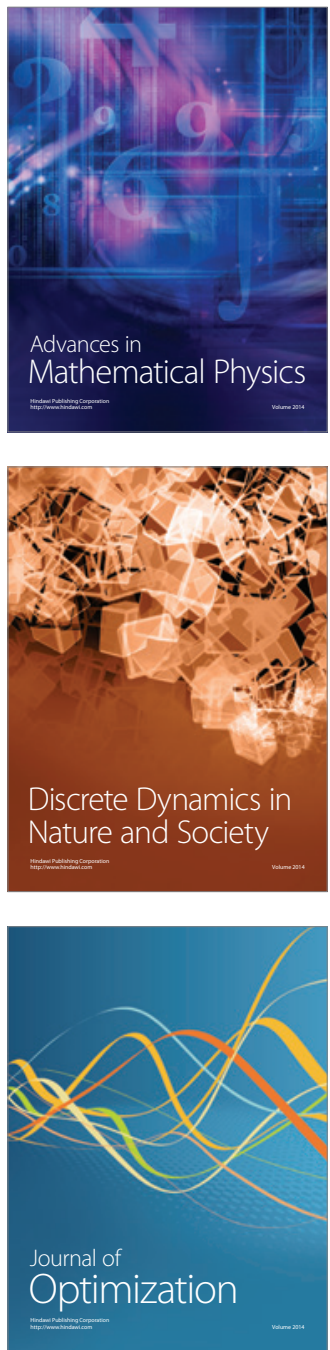